\title{
El proceso de descubrimiento en la clase de matemáticas: los razonamien- tos abductivo, inductivo y deductivo
}

\section{Discovery process in mathematics class: abductive, inductive and deductive reasoning}

\author{
María Nubia Soler-Álvarez \\ Universidad Pedagógica Nacional \\ nsoler@pedagogica.edu.co \\ Viviana Helena Manrique \\ vmanriqueperez@gmail.com
}

RESUMEN • En el presente artículo se exponen y analizan algunos resultados de una investigación desarrollada en la Universidad Pedagógica Nacional durante los años 2010 y 2011 (DMA-204-10). En esta investigación se caracterizan las diferentes formas de razonar usadas por un grupo de profesores de matemáticas en formación al resolver algunas actividades relacionadas con los números racionales e irracionales. El enfoque teórico usado para el desarrollo de la investigación retoma algunos aspectos de la teoría de Peirce sobre el método científico y sobre el razonamiento matemático, los cuales permiten interpretar las argumentaciones desarrolladas por los alumnos en la resolución de las diferentes actividades y discutir los resultados de otros estudios que se adscriben a la misma línea de investigación, como por ejemplo la propuesta de Cańadas y Castro (2007) sobre el proceso de conjeturación. Como resultado de este trabajo se obtiene la caracterización y clasificación de las diferentes formas de razonar empleadas en el proceso de descubrimiento matemático, así como algunos ejemplos de cada una de estas.

PALABRAS CLAVE: abducción; deducción; inducción; modelo de Toulmin; descubrimiento matemático.

\begin{abstract}
In this paper, we present and analyze some results of a research carried out at the Universidad Pedagógica Nacional during the years 2010 and 2011. In this study we characterize the different forms of reasoning used by a group of prospective mathematics teachers to address some activities related to rational and irrational numbers. The theoretical approach used for the development of research incorporates some aspects of Peirce's theory of scientific method and mathematical reasoning, which can interpret the arguments developed by students in the resolution of the different task and discuss the results of other studies that are assigned to the same line of research, such as the proposed Cañadas and Castro (2007) about conjecture process. As a result of this work we obtain the characterization and classification of different forms of reasoning employed in the process of mathematical discovery and examples of each of these.
\end{abstract}

KEYWORDS: abduction; deduction; induction; Toulmin model; mathematics discovery

Fecha de recepción: octubre 2012 • Aceptado: junio 2013

Manrique, V.H., Soler-Álvarez, M.N. (2014) El proceso de descubrimiento en la clase de matemáticas: los razonamientos abductivo, inductivo y deductivo. Enseñanza de las Ciencias, 32 (2), pp. 191-219 


\section{INTRODUCCIÓN}

El ministerio de Educación Nacional de Colombia -MEN- en los lineamientos curriculares establece que la educación matemática en los diferentes niveles, especialmente en básica y media, requiere que en el aula de matemáticas el maestro genere ambientes para que los estudiantes desarrollen actividad matemática (MEN, 1998). De esta propuesta del MEN surgen algunas preguntas, como por ejemplo, ¿qué se está entendiendo por actividad científica?, ¿es diferente la actividad científica de la matemática?, ¿cómo se puede desarrollar la actividad matemática en el aula?, ¿cómo se caracteriza la actividad matemática en el aula?, ¿qué tareas en el aula desarrollan algunos aspectos de la actividad científica?, entre otras. El presente trabajo se suscribe a los intereses del Ministerio de Educación Nacional y pretende dar respuesta a algunas de estas preguntas desde la formación de profesores de matemáticas, entendiendo que para que la actividad matemática ocurra en el aula es necesario que el maestro se forme en un ambiente en el que sean fundamentales el conocimiento profundo de los objetos matemáticos, la argumentación y, principalmente, el hacer matemáticas.

Teniendo de presente estas ideas, durante los años 2010 y 2011 se realizó en el Departamento de Matemáticas de la Universidad Pedagógica Nacional el proyecto de investigación Actividades matemáticas para el desarrollo de procesos lógicos: los razonamientos inductivo y abductivo (Dma-204-10). Este proyecto tenía como propósito identificar y caracterizar los razonamientos desarrollados por los estudiantes del curso de Sistemas numéricos del segundo semestre de 2010. Se estudiaron los desarrollos logrados por los estudiantes al realizar tareas relacionadas con conjuntos numéricos, como los fraccionarios, los racionales positivos y los irracionales positivos. Asociado a esta investigación se realizaron dos trabajos de grado (Nitola y Castańo, 2011; Romero y Parra, 2011), que tenían el mismo propósito, y un trabajo de grado (Manrique, 2012), en el que se identificaron y caracterizaron razonamientos desarrollados por estudiantes de último semestre de la licenciatura de Matemáticas al realizar una tarea sobre ruletas cicloidales, usando software de matemática dinámica. ${ }^{1}$

Como resultado de esta investigación, se presenta este artículo, el cual aporta respuestas a las preguntas enunciadas anteriormente describiendo el proceso de descubrimiento matemático en la clase de matemáticas a partir de ejemplos tomados de la investigación y de los trabajos de grado realizados. El proceso de descubrimiento matemático en la clase de matemáticas se puede resumir a partir del desarrollo de tres tipos de razonamientos: abductivo, inductivo y deductivo. El primer razonamiento, que tiene implícita la idea de la creatividad, apoya la formulación de conjeturas, y el segundo y tercer tipo de razonamiento apoyan la verificación y validación de conjeturas.

\section{REFERENTES TEÓRICOS}

Tal y como afirma Campos (2010), Charles Sanders Peirce dedicó gran parte de su obra al estudio de la lógica en la actividad matemática, formando parte de un grupo de filósofos que durante los siglos XIX y XX se dedicaron a estudiar la lógica de la investigación en las ciencias y especialmente en las matemáticas.

Aunque este autor consideraba que la cuestión de cómo los matemáticos proponen formas validas para resolver un problema matemático o para desarrollar la demostración de algún resultado matemático consiste en un "misterio" que requiere toda una vida de estudio, él mismo dedicó su vida al estudio de este misterio.

1. El término matemática dinámica, acuñado por el grupo de investigación Clase de Matemáticas, se refiere a programas que permiten el desarrollo de tareas asociadas a diferentes áreas de la matemática. A modo de ejemplo se encuentra el programa Geogebra, que brinda simultáneamente herramientas de geometría, álgebra, algebra lineal, cálculo, entre otras. 
El presente trabajo hace uso de algunos elementos de la teoría de Peirce sobre razonamiento para interpretar las acciones que se llevan a cabo en el descubrimiento de los estudiantes cuando realizan actividades propias de la clase de matemáticas. Para ello, se abordan dos enfoques de la teoría mencionada: las tres clases de razonar y la semiótica. Asimismo, se tiene en cuenta el modelo de Toulmin porque, como veremos, se corresponde con la teoría de Peirce y como herramienta metodológica permite diferenciar y caracterizar los conceptos inmersos en esta teoría.

\section{MODELO DE TOULMIN}

Para Toulmin (2003), a pesar de que los argumentos son producidos con una variedad de propósitos, la defensa formal de una afirmación es su fin primario. Según Chamizo (2007: 137),

... la mejor forma de considerar si una afirmación debe considerarse seriamente es revisando la base en la que se sustenta, desafiando sus credenciales y solicitando un argumento que la avale [...] como un argumento es el conjunto de razones que se dan a favor o en contra de una aseveración, cuando la afirmación es puesta en duda, la persona podrá apelar a los hechos y presentarlos para demostrar lo que ha dicho.

Este planteamiento se encuentra en la base del denominado modelo de Toulmin, el cual se presenta a continuación:

El modelo de argumentación que propone Toulmin (ver figura 1) consta de unos datos (D), una conclusión $(\mathrm{C})$, un garante $(\mathrm{G})$, un respaldo $(\mathrm{R})$, un cualificador $(\mathrm{Q})$ y unas refutaciones $(\mathrm{M})$.

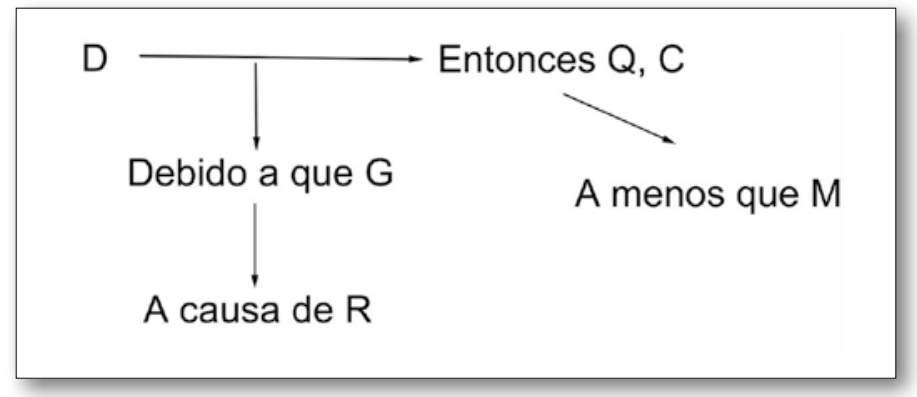

Fig. 1. Esquema del modelo de Toulmin sobre argumentación.

Mediante este modelo se dice que un argumento tiene lugar cuando a partir de unos hechos (datos) se elabora una afirmación (conclusión). Las proposiciones que justifican el paso de los datos a la conclusión se denominan garantes y, generalmente, hacen referencia a una regla, norma o principio general. El garante, a su vez, se sustenta en un grupo de afirmaciones que forman parte de un conjunto de contenidos o creencias denominado respaldo. Las refutaciones son el conjunto de circunstancias en las cuales el garante se podría anular y el cualificador es una construcción lingüística que acompaña a la conclusión, atenuándola. Estos últimos, refutaciones y cualificador, son de uso poco frecuente.

Aunque la teoría de Toulmin es usada ampliamente en el ámbito escolar para desarrollar argumentación en ciencias (ver Sardá y Sanmartí 2000), consideramos que es importante también su aplicación en el contexto de la enseńanza y el aprendizaje de las matemáticas, ya que como afirman estas autoras

El modelo de Toulmin, adaptado a la práctica escolar, permite reflexionar con el alumnado sobre la estructura del texto argumentativo y aclarar sus partes, destacando la importancia de las relaciones lógicas que debe haber entre ellas. Es decir, posibilita una metarreflexión sobre las características de una argumentación científica, profundizando sobre cómo se establecen las coordinaciones y las subordinaciones, sobre el uso de los diferentes tipos de conectores (adversativos, causales, consecutivos...), sobre la no-linealidad de los razonamientos, etc. 


\section{RAZONAMIENTO}

La teoría de Peirce acerca del razonamiento se distingue por su carácter evolutivo. A lo largo de su obra este autor manifiesta diversas posiciones acerca de lo que es razonar de forma inductiva, abductiva o deductiva; posiciones que él mismo fue reevaluando, sometiendo su trabajo a un constante proceso de autocrítica y autocorrección (Santaella, 2009). Como resultado de este proceso, la propuesta teórica de Peirce acerca del razonamiento se divide en dos etapas fundamentales, separadas por un periodo de transición.

Para los propósitos del presente documento se hará referencia a la segunda etapa de desarrollo de la teoría de Peirce, que comprende los documentos elaborados entre 1891 y 1914. Cabe señalar que esta segunda etapa no consiste en una teoría diferente a la planteada en la primera, sino que surge de la ampliación y depuración de los conceptos allí trabajados y que, por lo tanto, proporciona una concepción más elaborada de lo que son las tres formas de razonar.

En esta etapa Peirce define el razonamiento como el proceso mediante el cual se pasa de unas premisas a unas conclusiones por medio de un hábito general de pensamiento, que, aunque muchas veces no es reconocido por el razonador, es el que este considera que conduce al conocimiento verdadero, en el que no hay dudas. Obsérvese que un razonamiento es un tipo especial de argumento de acuerdo con el modelo de Toulmin, descrito líneas atrás.

Para Peirce, el razonamiento es de tres tipos: abductivo, inductivo y deductivo. Lo que hay que destacar es que, en esta etapa, las tres formas de razonar coexisten como tres estadios interdependientes y entrelazados del método científico, definidos de la siguiente manera:

La abducción se da cuando a partir de unos hechos, que se pueden considerar como sorprendentes y que demandan una explicación, se adopta una teoría, expresada en términos de hipótesis, la cual en caso de ser verdadera implica la verdad de los hechos.

Después de que una teoría es adoptada, se trazan sus probables y necesarias consecuencias; este paso lo denomina razonamiento deductivo. Habiendo extraído por medio de la deducción las predicciones de una teoría con relación a los que serían los resultados de un experimento, se procede a probarla haciendo los experimentos y comparando aquellas predicciones con los resultados reales del experimento; este proceso se denomina razonamiento inductivo.

Desde esta perspectiva, la inducción tiene un carácter comprobatorio y no creador, como se considera en muchos casos, es decir, los razonamientos inductivos no aportan conocimiento nuevo; su función es la de verificación de la teoría y, en algunos casos, de modificación de esta. Su principal labor es la de ir buscando el carácter de verdad de la teoría planteada.

La abducción es el razonamiento que le proporciona al razonador la teoría que la inducción verifica; al abducir, el razonador estudia un fenómeno para él sorprendente, examina sus características e identifica algunas relaciones entre estas y sugiere una teoría que explica lo que es sorprendente del fenómeno. La abducción, por lo tanto, corresponde al razonamiento que permite generar nuevas ideas, es donde se evidencia un proceso creativo en la generación de conocimiento.

Los razonamientos abductivo e inductivo son formulados inicialmente por Peirce como parte del método científico, pero revisando en detalle sus definiciones estos también se utilizan en la creación de teorías matemáticas: la abducción induce una generalización y la inducción confirma de manera probable dicha generalización. Hay que tener en cuenta que estos dos razonamientos, en el caso de las matemáticas, no se presentan en el mundo real, sino en el estado hipotético de las cosas, luego la experimentación no se haría sobre fenómenos del mundo real, sino sobre los diagramas matemáticos (Campos, 2010).

Una de las principales dificultades para aceptar estos dos tipos de razonamiento en la generación de conocimiento matemático radica en que la inducción no sería suficiente para aceptar o validar la teoría 
propuesta, este último tipo de razonamiento serviría para ir acercándose a la verdad de la teoría o para ir depurándola. Para el método matemático, la validación de una teoría surgida en un razonamiento abductivo estaría centrada entonces en el razonamiento deductivo, el cual debe garantizar que, de los datos iniciales, se siga necesariamente la verdad de la teoría.

\section{SEMIÓTICA}

Según señala Campos (2010), en la teoría de Peirce un signo es algo que representa a alguna cosa por otra cosa. El signo se constituye a través de la relación triádica entre el objeto, el signo y el intérprete. Las relaciones entre los signos y los objetos, mediadas por el intérprete, se clasifican en tres tipos: símbolos, índices e iconos. El símbolo significa el objeto por convención. El índice significa el objeto por estar realmente conectado a este. Y el icono significa al objeto por semejanza.

El razonamiento matemático es conducido a través de iconos matemáticos, también denominados diagramas matemáticos. Un icono matemático es aquel signo que da cuenta de todas las propiedades del objeto matemático que representa, mientras que un diagrama es una construcción mental de un objeto, susceptible de cambio. En general, un diagrama matemático es una clase de icono que representa en la mente las relaciones que se involucran en una hipótesis.

Un diagrama geométrico, como el que nos imaginamos para representar un triángulo por ejemplo, es una clase de icono matemático, en el cual un conjunto ordenado de puntos y líneas representa al objeto geométrico, mientras que un diagrama algebraico, como una ecuación, es una unión de letras y otros caracteres que representan un objeto.

El proceso de razonar matemáticamente desde la perspectiva de Peirce tiene algunos puntos de encuentro con lo presentado anteriormente sobre las tres formas de razonar en ciencias según este mismo autor. El razonamiento matemático empieza cuando en la imaginación se forma alguna clase de representación de hechos o fenómenos observados. Esta representación se hace a partir de diagramas, los cuales adicionalmente permiten identificar las relaciones encontradas en los hechos observados. Cada diagrama es observado y de este se sugiere una hipótesis que describe los hechos (razonamiento abductivo). Luego se procede a experimentar sobre el diagrama (razonamiento inductivo) y a transformarlo con el fin de validar dicha hipótesis (razonamiento deductivo). El conocimiento matemático emerge de las hipótesis validadas a través de este proceso.

\section{EL PROCESO DE DESCUBRIMIENTO EN MATEMÁTICAS}

Para muchos autores y matemáticos, el desarrollo del conocimiento matemático se ha dado gracias a las conjeturas. Los matemáticos desde los tiempos antiguos han estado formulando conjeturas y buscando la manera de validarlas. En este sentido, el proceso de conjeturar debe estar presente como parte de la actividad matemática que se desarrolle en el aula de clase.

Cañadas y Castro (2007) describen el razonamiento inductivo, que es entendido de manera diferente a la planteada por Peirce, como el proceso de elaboración y verificación de conjeturas. Estos autores describen siete etapas en este proceso: i) observación de casos particulares, ii) organización de casos particulares, iii) búsqueda y predicción de patrones, iv) formulación de conjeturas, v) justificación de conjeturas basándose en casos particulares, vi) generalización y vii) demostración (justificación del caso general).

Haciendo una correspondencia entre lo planteado por Peirce y por Cañadas y Castro (2007), y utilizando el modelo de Toulmin, se observa que la estructura del razonamiento abductivo se corresponde con los pasos uno al cuatro establecidos por Cañadas y Castro. La observación y organización de los 
casos particulares corresponden a los datos del razonamiento; la búsqueda y predicción de patrones, al garante, y la formulación de conjeturas, a la conclusión. El esquema de este razonamiento se presenta en la figura 2.

\begin{tabular}{|c|c|c|c|}
\hline \multicolumn{4}{|c|}{ Razonamiento abductivo } \\
\hline D: & $\begin{array}{l}\text { Datos presentados en diferente } \\
\text { diagramas. }\end{array}$ & 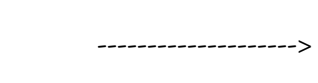 & C: Conjeturas \\
\hline & G: & \multicolumn{2}{|c|}{$\begin{array}{l}\text { Patrones, relaciones, regularidades, otras conjeturas y } \\
\text { propiedades observadas en los datos. }\end{array}$} \\
\hline & $\mathrm{R}:$ & \multicolumn{2}{|c|}{$\begin{array}{l}\text { Se sustenta en la definición de Peirce sobre razona- } \\
\text { miento abductivo: } \\
\text { La teoría formulada es probablemente cierta si los } \\
\text { datos estudiados se deducen necesariamente de esta. }\end{array}$} \\
\hline
\end{tabular}

Fig 2. Esquema general de un razonamiento abductivo.

El paso v, justificación de conjeturas con base en casos particulares, se corresponde con el razonamiento inductivo y el vii, validación de conjeturas, con el razonamiento deductivo. En los razonamientos deductivos, según Peirce, los hechos presentados en las premisas no pueden ser verdaderos bajo ninguna circunstancia, sin implicar la verdad de la conclusión. En la figura 3 se presenta el razonamiento deductivo en términos del modelo de Toulmin.

\begin{tabular}{|l|l|l|l|}
\hline \multicolumn{4}{|c|}{ Razonamiento deductivo } \\
\hline D: Premisa & \multicolumn{1}{|c|}{ C: Conclusión } \\
\hline & G: $\quad \begin{array}{l}\text { Teoremas, lemas, definiciones, proposiciones, reglas, } \\
\text { etc. }\end{array}$ \\
\cline { 2 - 3 } & $\begin{array}{l}\text { Lógicas de primer y segundo orden. } \\
\text { R: } \\
\text { Contexto matemático en el que se presenta la con- } \\
\text { jetura. }\end{array}$ \\
\hline
\end{tabular}

Fig 3. Esquema general de un razonamiento deductivo.

El razonamiento inductivo ${ }^{2}$ se da cuando el estudiante realiza experimentaciones para determinar si la conjetura planteada es verdadera. El esquema general de un razonamiento inductivo se presenta en la figura 4. Cabe aclarar aquí que en matemáticas el razonamiento inductivo no garantiza la veracidad de las conjeturas, pero los estudiantes en su actividad validan sus conjeturas por medio de este tipo de razonamiento en un sinnúmero de ocasiones. Asimismo, es importante resaltar que programas de ma-

2. El razonamiento inductivo desde la perspectiva de Peirce, como se interpreta en este documento, es diferente al método de inducción matemática utilizado para demostrar propiedades sobre los números naturales teniendo como principio los axiomas de Peano. Sin embargo, se puede observar que tanto el razonamiento inductivo como el método de inducción se encuentran relacionados tal como se describe a continuación: Cuando se identifica una propiedad que podría ser válida para los números naturales, lo que generalmente se hace es verificar la propiedad en muchos y diferentes ejemplos. Hacer esta verificación es lo mismo que razonar de forma inductiva, desde la perspectiva de Peirce. Después de que la propiedad se verifica para estos ejemplos, se busca probar que es verdadera a través de una demostración por inducción. Esta demostración empieza verificando la propiedad para el primer elemento, luego, asumiendo que la propiedad es válida para un número cualquiera, se demuestra que es válida para el siguiente número. Esta demostración lo que hace es tomar un ejemplo cualquiera y garantizar que en este la propiedad se cumple, es decir, el método de inducción generaliza el razonamiento inductivo en el caso de los números naturales. 
temática dinámica, como Cabri o Geogebra, apoyan de forma significativa este tipo de razonamiento, dado que permiten estudiar un número considerable de casos de manera relativamente rápida.

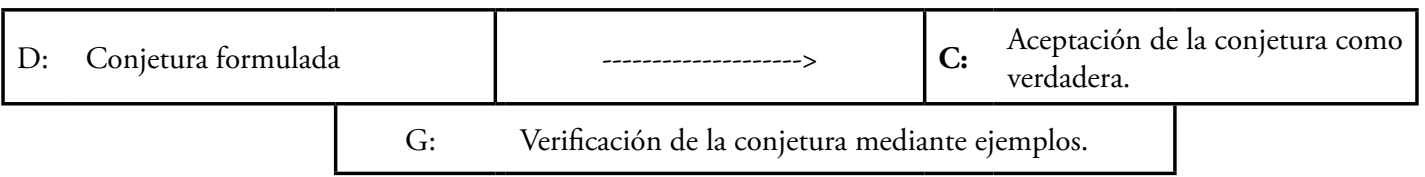

Fig. 4. Esquema general de un razonamiento inductivo.

\section{DISCUSIÓN}

A partir de lo expuesto hasta ahora, el proceso de descubrimiento en la clase de matemáticas se podría caracterizar a partir de las tres formas de razonar propuestas por Peirce: razonamiento abductivo, con el cual se producen conjeturas; razonamiento inductivo, en el que se verifican de forma experimental algunas conjeturas, y razonamiento deductivo, con el que se validan conjeturas. En lo que sigue se describen en detalle cada uno de estos razonamientos y se dan ejemplos tomados de la investigación realizada.

\section{Razonamiento abductivo-formulación de conjeturas}

En la formulación de conjeturas se distinguen tres momentos fundamentalmente: estudio de casos; observación de patrones, regularidades o propiedades, y presentación de conjeturas o conclusiones. El estudio de casos hace referencia a la manipulación y transformación de diferentes diagramas, sobre los cuales es posible luego observar regularidades o relaciones. En la observación de patrones, regularidades o propiedades, los estudiantes identifican lo que es común en los diagramas estudiados. La formulación de conjeturas hace referencia a las diferentes formas en que los estudiantes las expresan: a través de ejemplos, de forma verbal, por medio de diagramas de diferente tipo o combinando estas formas.

Cañadas, Deulofeu, Figueras, Reid y Yevdokimov (2008) presentan una tipología de las conjeturas que pueden presentarse en un razonamiento abductivo; este artículo amplía la caracterización hecha por estos autores y la expresa en términos de la teoría de Peirce sobre razonamiento. La tabla 1 presenta la equivalencia entre las dos teorías.

Tabla 1.

Clases de abducción en correspondencia con lo propuesto por Cańadas et al. (2008).

\begin{tabular}{|l|l|}
\hline \multicolumn{1}{|c|}{$\begin{array}{c}\text { Tipología de conjeturas elaborada } \\
\text { por Cañadas, Deulofeu, Figueras, Reid y Yevdokimov }\end{array}$} & $\begin{array}{c}\text { Tipología en términos } \\
\text { del razonamiento abductivo según Peirce }\end{array}$ \\
\hline $\begin{array}{c}\text { Tipo 1: Inducción empírica a partir de un número finito de } \\
\text { casos discretos }\end{array}$ & Abducción de patrones \\
\hline Tipo 2: Inducción empírica a partir de casos dinámicos & Abducción con diagramas dinámicos \\
\hline Tipo 3: Analogía & Abducción por analogía \\
\hline Tipo 4: Abducción & $\begin{array}{l}\text { Abducción con base en una característica observada en un } \\
\text { caso particular }\end{array}$ \\
\hline Tipo 5: Basadas en la percepción & $\begin{array}{l}\text { Abducción con base en relaciones observadas en un diagra- } \\
\text { ma geométrico }\end{array}$ \\
\hline
\end{tabular}




\section{ABDUCCIÓN DE PATRONES}

Este tipo de abducción se deriva del primer tipo de conjeturas definido por Cañadas et al. (2008): inducción empírica a partir de un número finito de casos discretos. Esta se elabora sobre el reconocimiento de patrones observados en un conjunto finito de casos discretos. En los términos de la teoría de Peirce, este proceso se asocia a una abducción en la que el estudiante observa un patrón en un conjunto de diagramas numéricos y elabora una conjetura que generaliza el patrón observado. Usando el esquema de Toulmin, este tipo de abducción tiene la estructura propuesta en la figura 5.

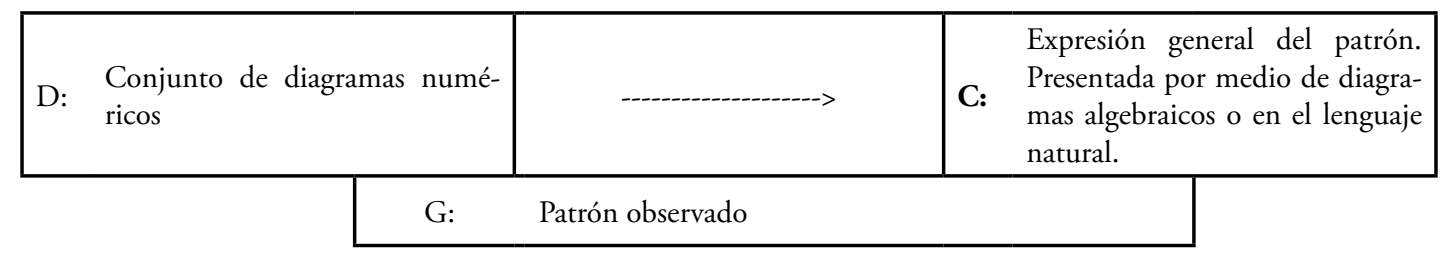

Fig. 5. Esquema razonamiento "Abducción de patrones"

Algunos ejemplos de este tipo de abducción se observaron en el desarrollo de las tareas denominadas "Fracciones a racionales" y "Fraccionarios", realizadas con estudiantes del curso de Sistemas numéricos de la licenciatura de Matemáticas de la Universidad Pedagógica Nacional en el segundo semestre de 2010 (Nitola y Castańo, 2011; Romero y Parra, 2011). También se observa este tipo de abducción en el desarrollo de la tarea "Epicicloides e hipocicloides", realizada por estudiantes de últimos semestres de la licenciatura y reportada en el trabajo de Manrique (2012).

\section{Ejemplo en la tarea "Fracciones a racionales"}

La tarea "Fracciones a racionales" consistía en encontrar una correspondencia entre las fracciones representadas en el plano cartesiano con los números naturales, a partir de la posición que estas ocupan siguiendo el orden propuesto en la figura 6 . En otros términos, los estudiantes debían decir dada la posición qué fracción se encontraba representada allí y viceversa.

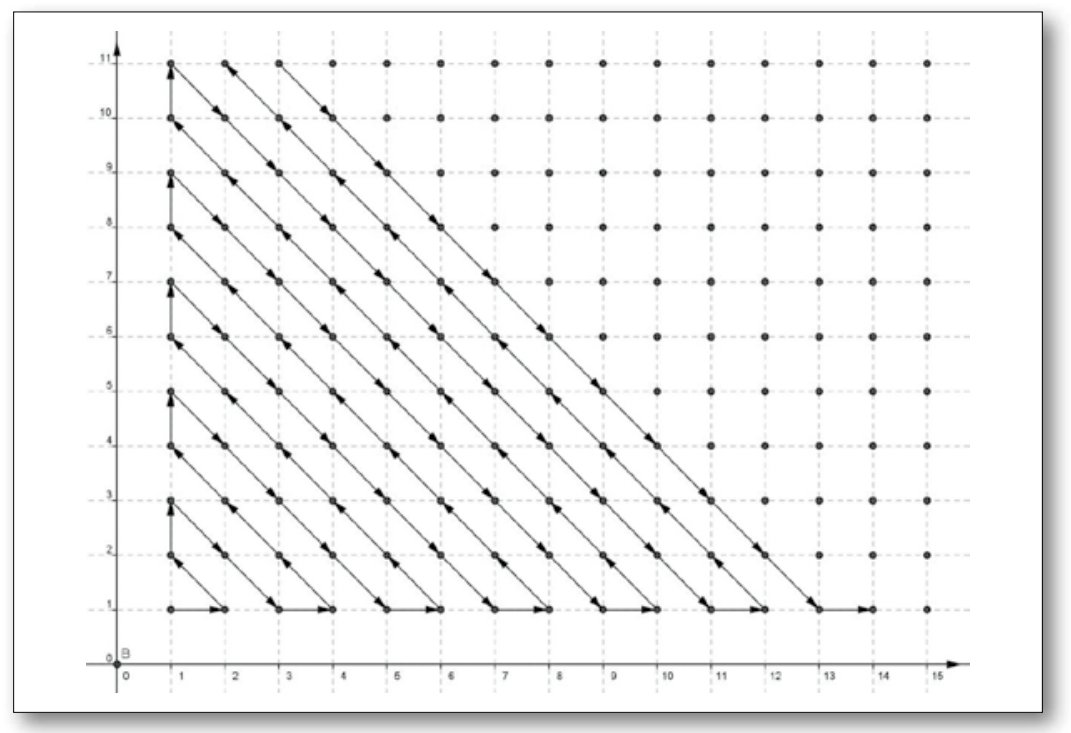

Fig. 6. Representación cartesiana de las fracciones y método para contar fraccionarios 
Tomando algunos casos particulares, los estudiantes lograron encontrar un patrón relacionado con los números triangulares que permitía determinar la posición de algunas fracciones. La figura 7 presenta el razonamiento logrado por los estudiantes.

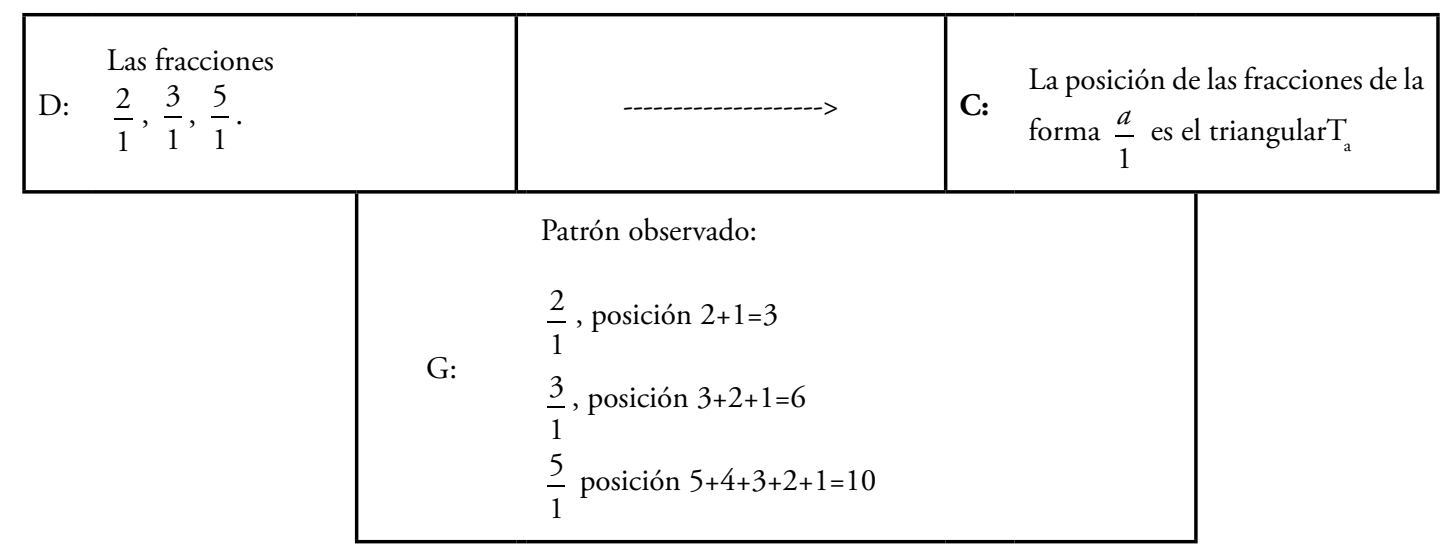

Fig. 7. Abducción de patrones en el desarrollo de la tarea "Fracciones a racionales"

Después de la formulación de esta conjetura, los estudiantes encontraron que era falsa, dado que observaron que la posición de la fracción $\frac{4}{1}$ no era un número triangular, es decir, desarrollaron un
contraejemplo.

\section{Ejemplo en la tarea "fraccionarios"}

Esta tarea pretendía que los estudiantes reconocieran e identificaran relaciones entre diferentes representaciones de los números fraccionarios. Dos representaciones son la numérica usual y la de fracción continua simple. La tercera representación, tomada de Redondo (2005), es una forma gráfica particular de las fracciones en la que se utilizan cuadrados máximos inscritos en un rectángulo. Los cuadrados considerados en esta representación corresponden a los coeficientes de la fracción continua finita y las dimensiones de este, a la representación numérica usual.

La tarea consistía en entregarle a los estudiantes tablas a tres columnas en las que se presentaban ejemplos de fracciones representadas de las tres maneras, se les pedía que establecieran relaciones entre las columnas y que justificaran dichas relaciones con ejemplos y de manera general (ver tabla 2). Esta tarea descrita de manera amplia y su desarrollo se encuentra detallada en Romero y Parra (2011).

3. En términos de modelo de Toulmin, encontrar un contraejemplo corresponde a un razonamiento de tipo deductivo en el que los datos corresponden a "la fracción $\frac{4}{1}$ no está ubicada en una posición triangular", la conclusión, a la afirmación "no es cierto que la posición de las fracciones de la forma $\frac{a}{1}$ es el triangular $\mathrm{T}_{\mathrm{a}}$ ” $\mathrm{y}$ el garante corresponde a la regla de inferencia $\exists y(p(y) \wedge \neg q(y)) \leftrightarrow \forall x(p(x) \rightarrow q(x))$. 
Observa la siguiente tabla y describe las relaciones que se dan entre las tres columnas:

Tabla 2.

Primera parte de la tarea "fraccionarios"

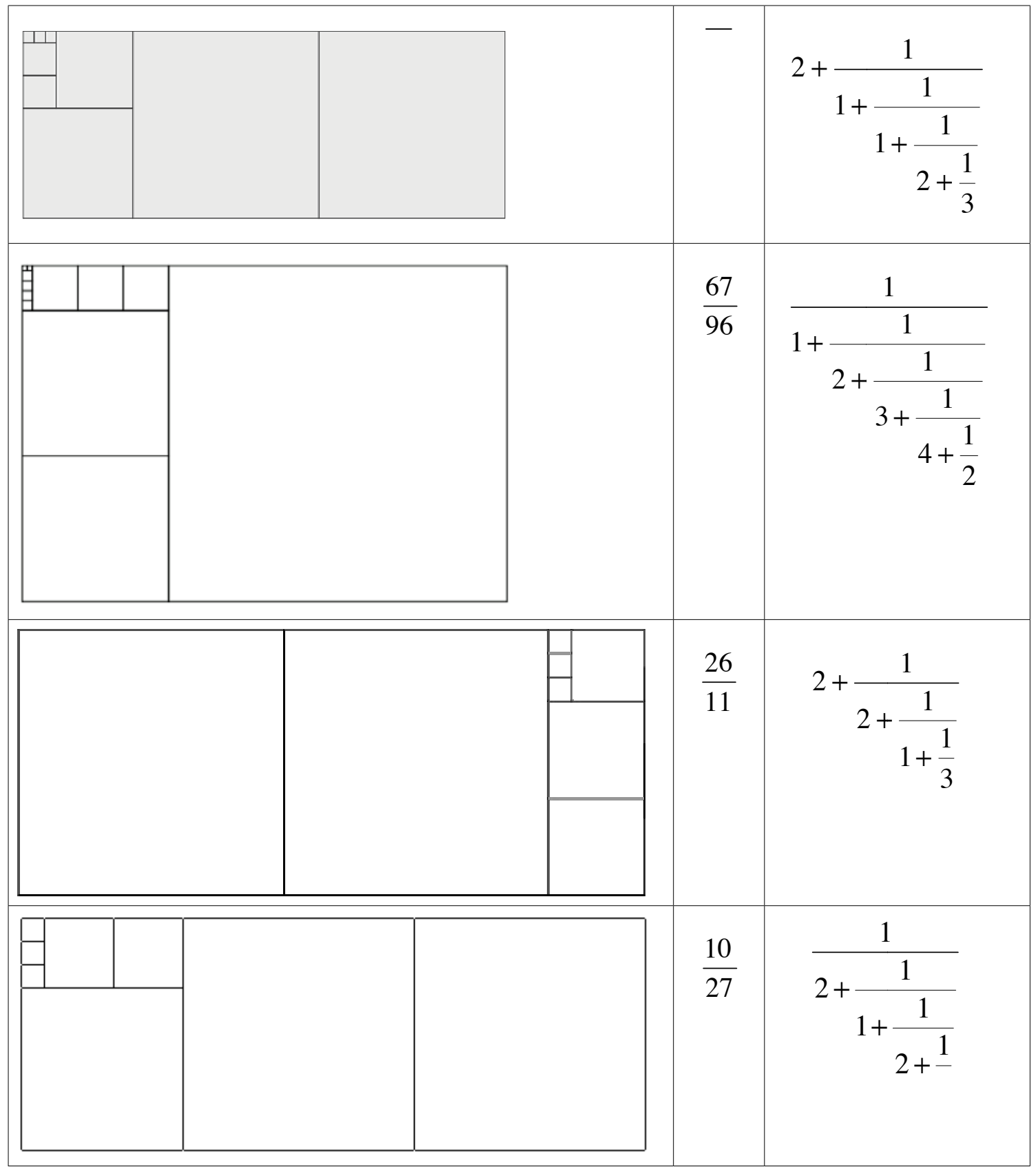

Los estudiantes desarrollaron abducción de patrones en esta tarea cuando representaron varias fracciones como una fracción continua simple finita y observaron que este procedimiento se podía desarrollar siempre que las fracciones no fueran de la forma. La figura 8 esquematiza este razonamiento. 


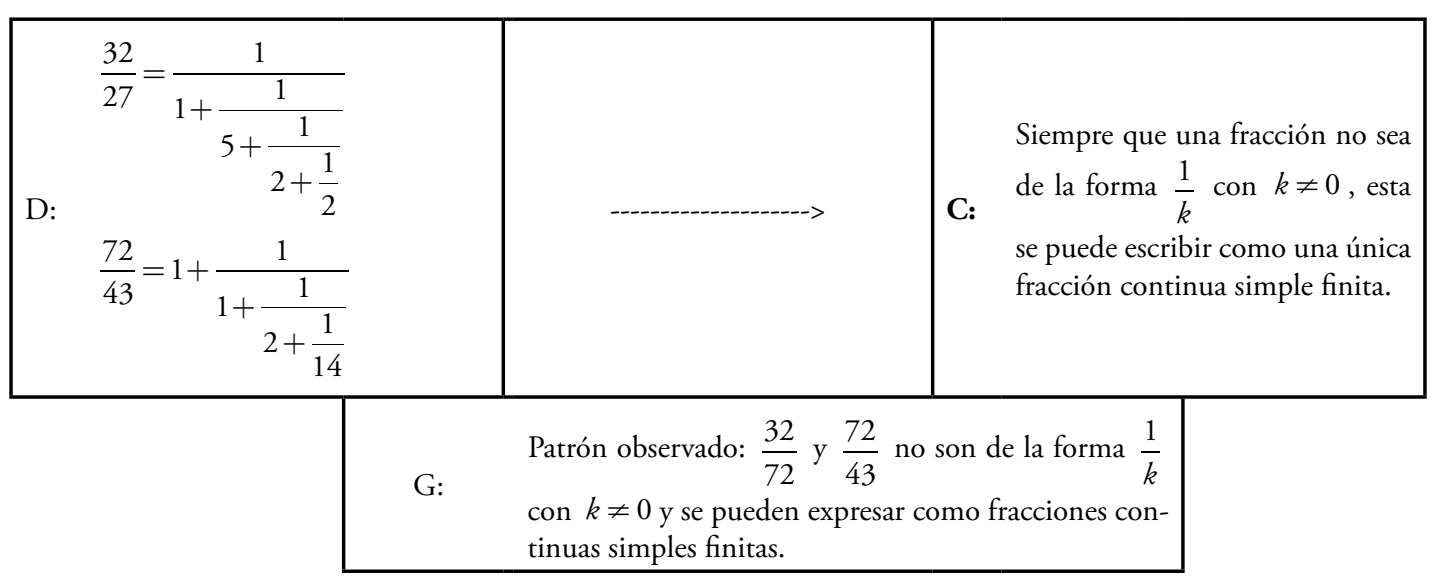

Fig 8. Abducción de patrones en el desarrollo de la tarea "Fraccionarios"

\section{Ejemplo en la tarea "Epicicloides e hipocicloides"}

Para esta tarea, los estudiantes -a partir de un applet en el que se pueden observar los diferentes tipos de cicloides e hipocicloides que se generan al cambiar los radios de las circunferencias generatriz y directriz- debían completar una tabla en la que se preguntaba por los radios de la generatriz y directriz ( $a$ y $b$ respectivamente), el número de vértices de la figura $(v)$ y el mínimo número de recorridos que da la circunferencia generatriz para obtener la curva $(r)$. Algunos pantallazos de este applet se pueden ver en la figura 12; la tabla 3 es la que los estudiantes debían completar. A partir de la tabla, los estudiantes formularon una conjetura en la que relacionaron los datos de esta. La figura 9 presenta el razonamiento abductivo logrado.

Tabla 3.

Datos dados en la tarea "Epicicloides e hipocicloides"

\begin{tabular}{|c|c|c|c|c|c|}
\hline Ruleta & $\begin{array}{c}\text { Radio } \\
\text { de la directriz (a) }\end{array}$ & $\begin{array}{l}\text { Radio } \\
\text { de la generatriz } \\
\text { (b) }\end{array}$ & $\begin{array}{c}\text { Cantidad } \\
\text { de vértices o arcos }\end{array}$ & $\begin{array}{c}\text { Cantidad de recorridos } \\
\text { de la generatriz sobre la } \\
\text { direcriz }\end{array}$ & Cociente $(\mathrm{a} / \mathrm{b})$ \\
\hline \multirow[t]{7}{*}{ Epicicloide } & 8 & 2 & & & \\
\hline & 4 & 2.5 & & & \\
\hline & & & 5 & 1 & \\
\hline & & & & 1 & 3 \\
\hline & 3 & & 1 & 2 & \\
\hline & & 6 & 1 & 5 & \\
\hline & & 1.8 & 10 & & \\
\hline \multirow[t]{8}{*}{ Hipocicloide } & 8 & 4 & & & \\
\hline & 8 & & 16 & 9 & \\
\hline & & 4.8 & & 2 & 2.5 \\
\hline & & & 10 & 3 & \\
\hline & 3 & & & 1 & 1 \\
\hline & 4.8 & & & & 1.6 \\
\hline & 7.7 & & & 1 & 7 \\
\hline & & & & & 1.8 \\
\hline
\end{tabular}


Tabla 4.

Datos encontrados por los estudiantes en la tarea "Epicicloides e hipocicloides"

\begin{tabular}{|c|c|c|c|c|}
\hline RULETA & $\mathrm{a}$ & $\mathrm{b}$ & $\mathrm{v}$ & $\mathrm{r}$ \\
\hline \multirow{2}{*}{ EPICICLOIDE } & 8 & 2 & 4 & 1 \\
\cline { 2 - 5 } & 3 & 6 & 1 & 2 \\
\hline \multirow{2}{*}{ HIPOCICLOIDE } & 8 & 4 & 2 & 1 \\
\cline { 2 - 5 } & 12 & 4.8 & 5 & 2 \\
\hline
\end{tabular}

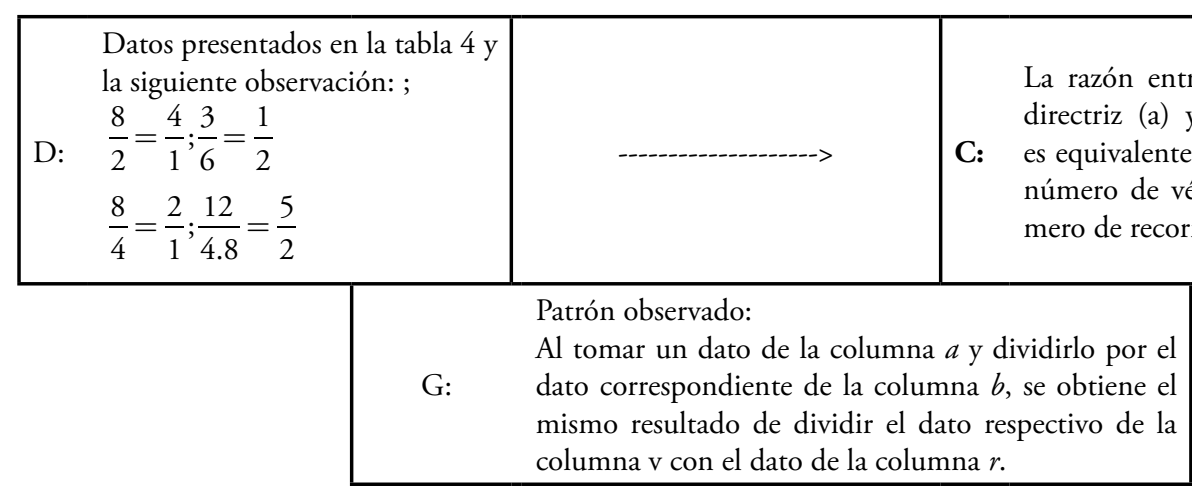

Fig 9. Abducción de patrones en el desarrollo de la tarea "Epicicloides e hipocicloides"

\section{ABDUCCIÓN CON DIAGRAMAS DINÁMICOS}

Esta clase de abducción se define con base en el segundo tipo de conjeturas definida por Cañadas et al. (2008): inducción empírica a partir de casos dinámicos. Según estos autores, esta conjetura se elabora sobre la observación de una propiedad invariante presente en un número infinito de casos continuos que son un subconjunto de un número infinito de casos posibles. Asociamos la elaboración de este tipo de conjetura a un proceso abductivo en el que la conjetura se establece a partir de construcciones geométricas o variables presentes en un contexto de matemática dinámica y obedece a la generalización de una propiedad observada en dichas construcciones o variables. Usando el esquema del modelo de Toulmin se obtendría la estructura presentada en la figura 10. Tareas apoyadas en programas de matemática dinámica, como las mencionadas anteriormente, permiten desarrollar de forma significativa los razonamientos abductivo e inductivo. En el ejemplo que se presenta en relación con la tarea "Epicicloides e hipocicloides" se observa cómo el entorno dinámico, junto con la formulación de preguntas abiertas, permite que los estudiantes visualicen múltiples relaciones entre objetos matemáticos y con esto formulen una gran cantidad de conjeturas.

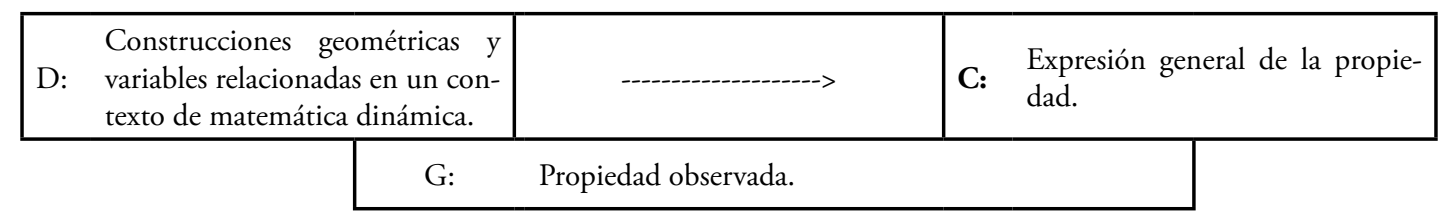

Fig. 10. Abducción a partir de diagramas dinámicos 


\section{Ejemplo en la tarea "Epicicloides e hipocicloides"}

De la observación de las curvas que se generaban en los applets, los estudiantes plantearon razonamientos abductivos; en la figura 11 se presenta uno de estos razonamientos.

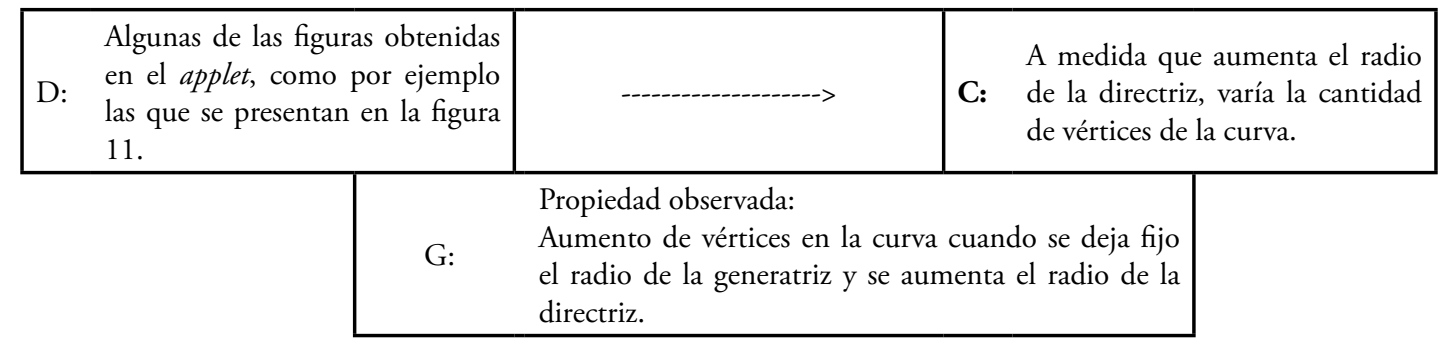

Fig. 11. Abducción a partir de diagramas dinámicos en la tarea "Epicicloides e hipocicloides"

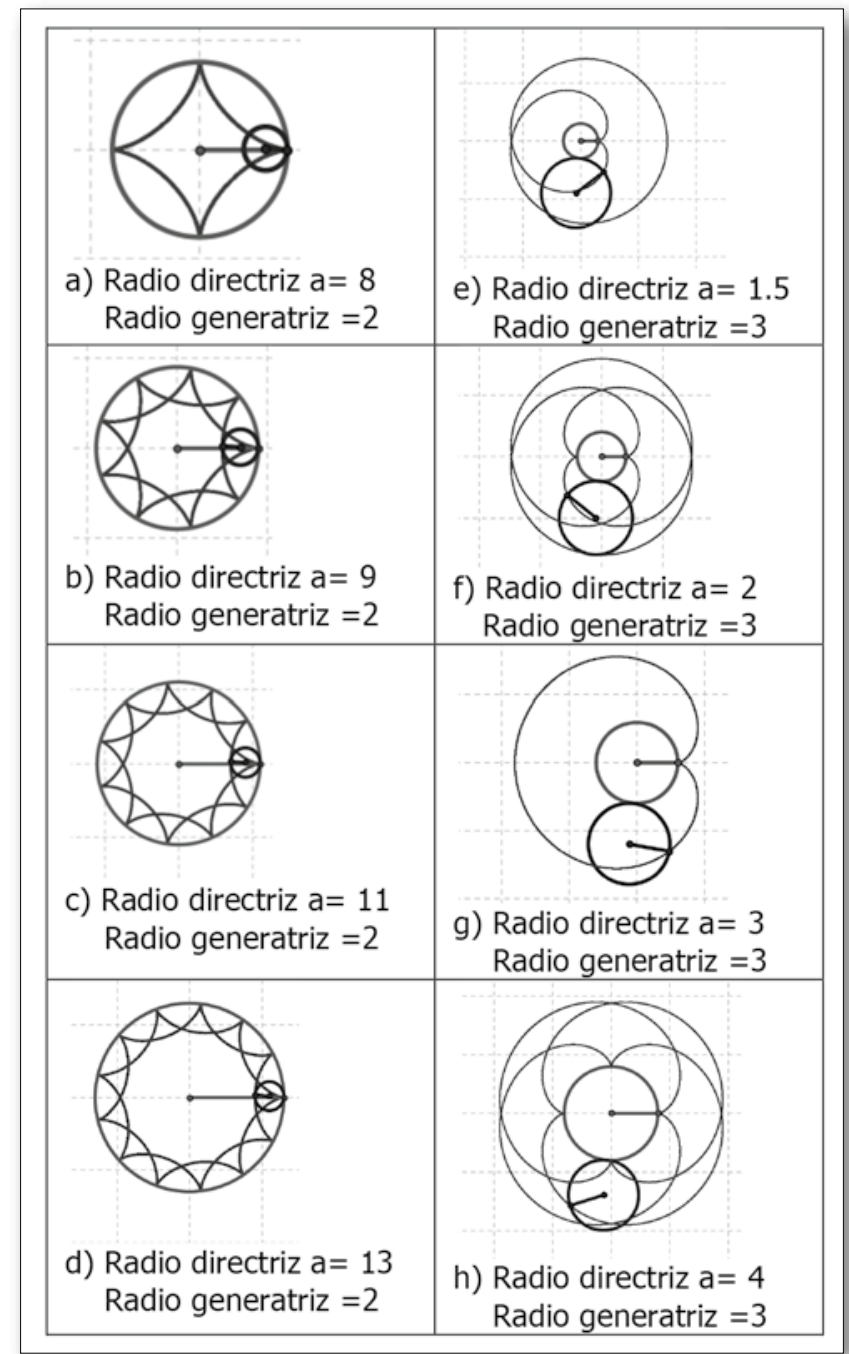

Fig. 12. Imágenes de los applets hipocicloide y epicicloide cuando se aumenta el radio de la recta directriz y se fija el radio de la generatriz 


\section{ABDUCCIÓN POR ANALOGÍA}

En los términos de Cañadas et al. (2008), este tipo de razonamiento se corresponde con el tipo de conjetura denominado de analogía, el cual se elabora sobre un hecho particular conocido o sobre la base de una regla general establecida previamente. Este proceso se asocia a un razonamiento abductivo en el que la conjetura se establece con base en una conjetura creada en una abducción previa o un hecho conocido anteriormente. Como conclusión, se obtiene la modificación de dicha conjetura para un nuevo caso, como se muestra en el esquema presentado en la figura 13.

\begin{tabular}{|l|c|c|c|c|}
\hline D: $\begin{array}{l}\text { Hecho conocido y caso que se } \\
\text { está estudiando. }\end{array}$ & C: Conjetura \\
\hline & G: $\quad \begin{array}{l}\text { Relación entre un hecho conocido o una abducción } \\
\text { previa y el caso que se está estudiando. }\end{array}$ & \\
\cline { 2 - 3 } & &
\end{tabular}

Fig. 13. Abducción a partir de una abducción previa

Un ejemplo de este tipo de conjetura se presentó en la realización de la tarea de suma y la multiplicación de fracciones.

\section{Ejemplo en la tarea "Suma y multiplicación de fracciones"}

La conjetura surge cuando los alumnos tienen como tarea inventarse un procedimiento gráfico para multiplicar fraccionarios que sea compatible con el algoritmo numérico usual. Los estudiantes recuerdan un método gráfico para multiplicar números naturales compatible con el método numérico y con base en este se inventan un procedimiento para multiplicar fracciones, logrando con esto el argumento que se presenta en la figura 14.

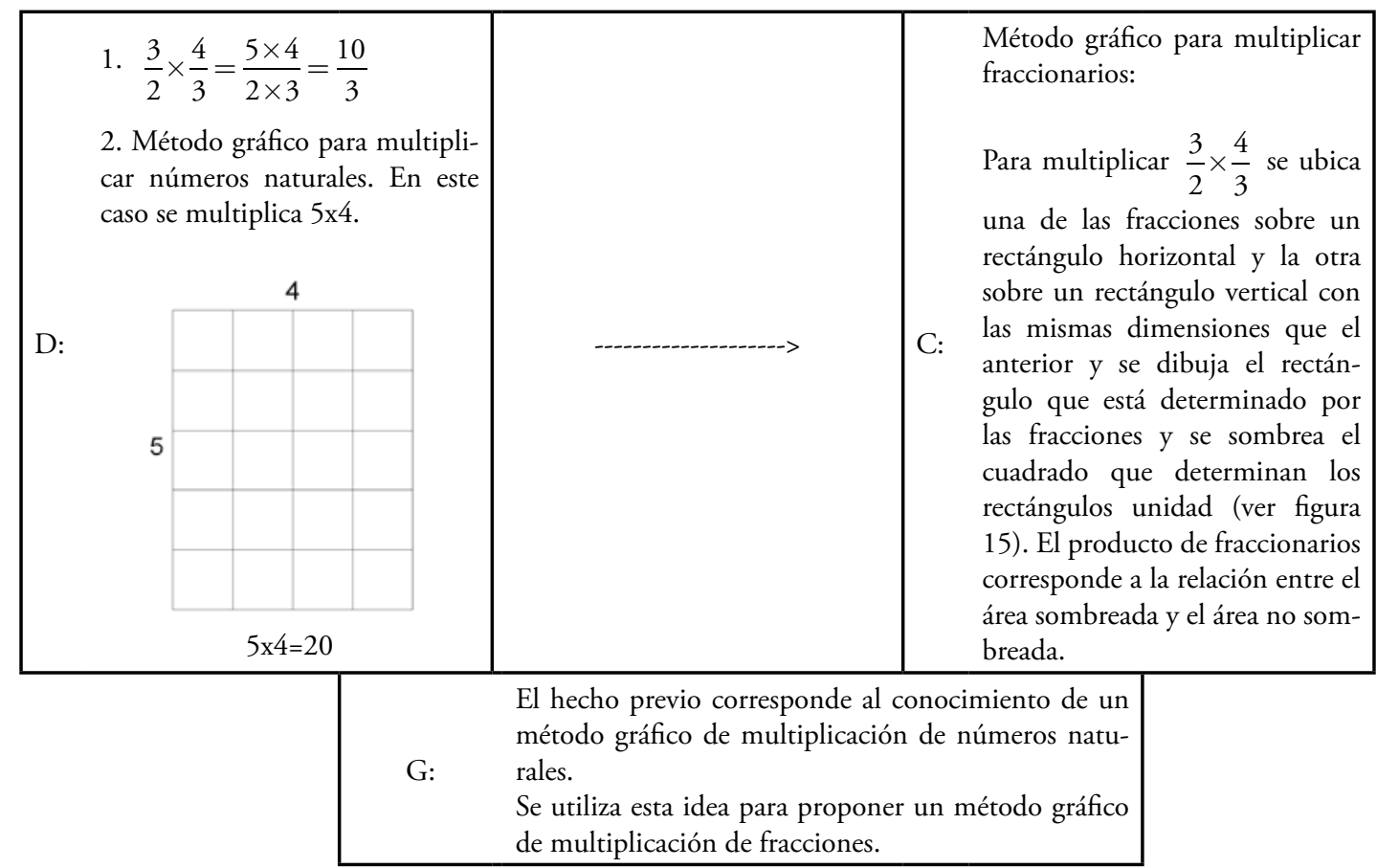

Fig. 14. Abducción con base en una abducción previa en la tarea suma de fracciones 


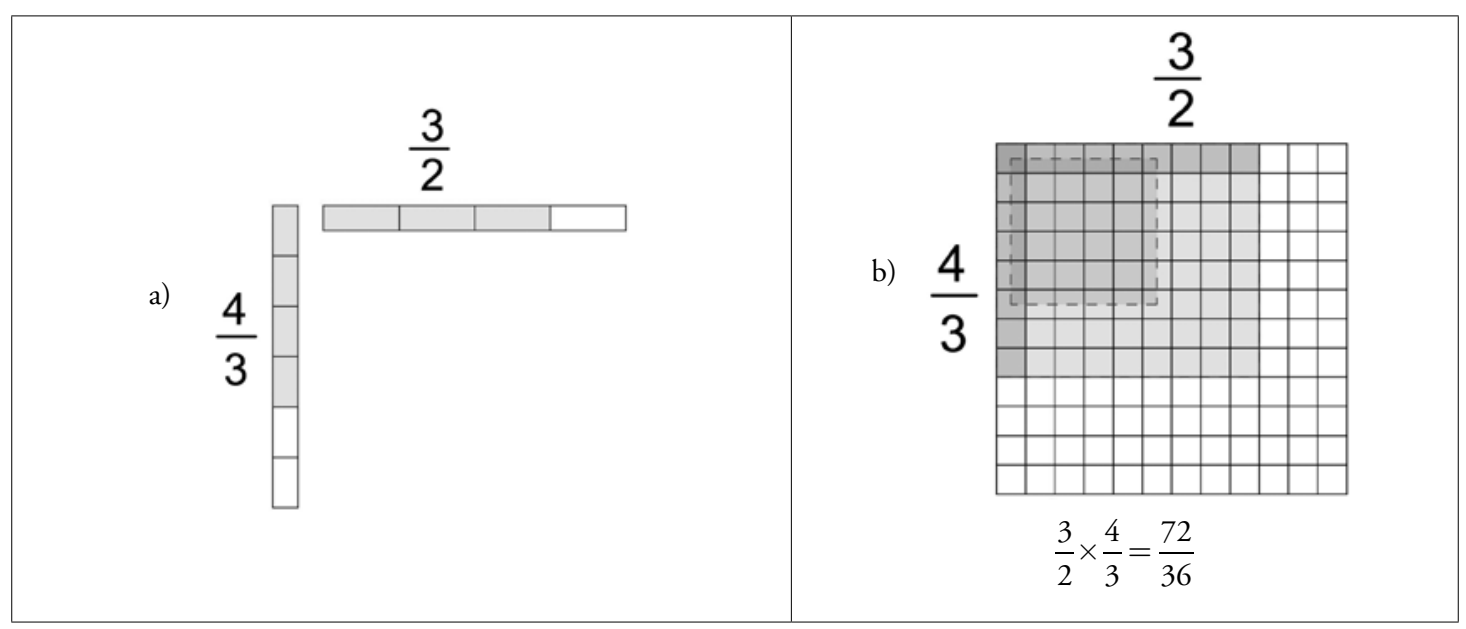

Fig. 15. Diagrama geométrico de un método gráfico de multiplicación de fracciones

\section{ABDUCCIÓN CON BASE EN UNA CARACTERÍSTICA OBSERVADA EN UN CASO PARTICULAR}

Cañadas y sus colaboradores definen un cuarto tipo de conjetura, al que denominan abducción porque se establece partiendo de un solo ejemplo o caso y surge como una regla general que permitirá explicar este acontecimiento, inexplicable de otra manera. Esta definición se corresponde con la concepción de abducción de Peirce en la primera etapa de desarrollo de su teoría. Consideramos que este proceso es una abducción en la que la conjetura tiene como base una característica observada en un caso particular y surge como una generalización de dicha característica. En consecuencia, este tipo de abducción es un caso particular del primer tipo de abducción (abducción de patrones), para el cual se considera un único caso. Usando el esquema de Toulmin, este tipo de abducción tiene las características presentadas en la figura 16.

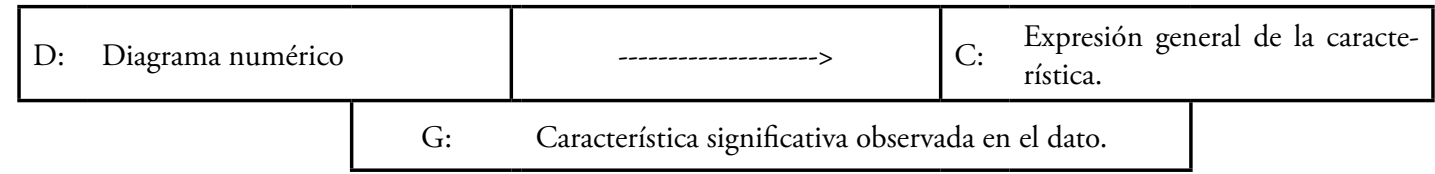

Fig. 16. Abducción con base en un caso particular

Algunas abducciones de este tipo se presentan en el desarrollo de las tareas "Fracciones a racionales" y "Fraccionarios".

\section{Ejemplo en la tarea "Fracciones a racionales"}

En el desarrollo de la tarea "Fracciones a racionales", en la plenaria, un estudiante presenta una fórmula para determinar la fracción ubicada en una posición. La fórmula es $F_{P}=\frac{j}{(n+1)-j+1}$, donde $K n$ es el mayor de los números triangulares menores que $P$ y $j=P-K_{n}$.

Los estudiantes desarrollan un ejemplo para verificar si la fórmula funciona y observan que esta sirve cuando $n$ es par, pero para $n$ impar, la fracción encontrada utilizando la fórmula es la inversa a la que se está buscando; con este ejemplo modifican la fórmula y desarrollan el razonamiento abductivo que se presenta en la figura 17. 


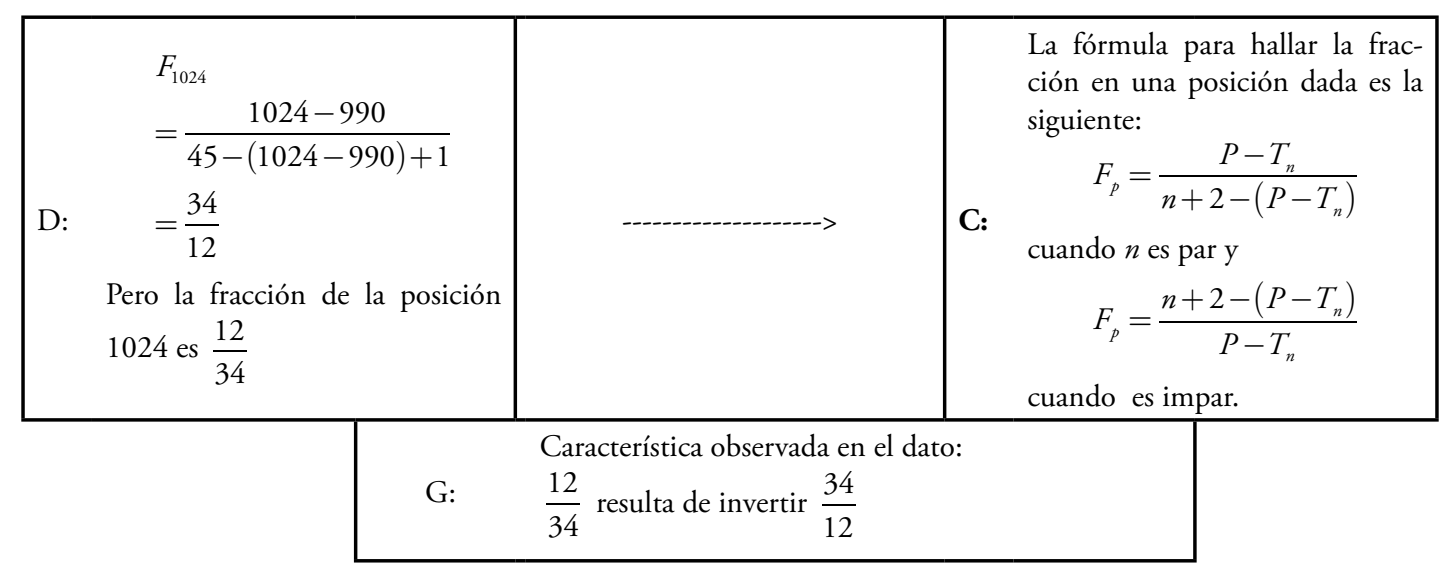

Fig. 17. Abducción con base en un caso particular en la tarea "Fracciones a racionales"

\section{Ejemplo en la tarea "Fraccionarios"}

La abducción tiene lugar cuando los alumnos están pasando de la fracción continua a la fracción en un caso particular (ver figura 18).

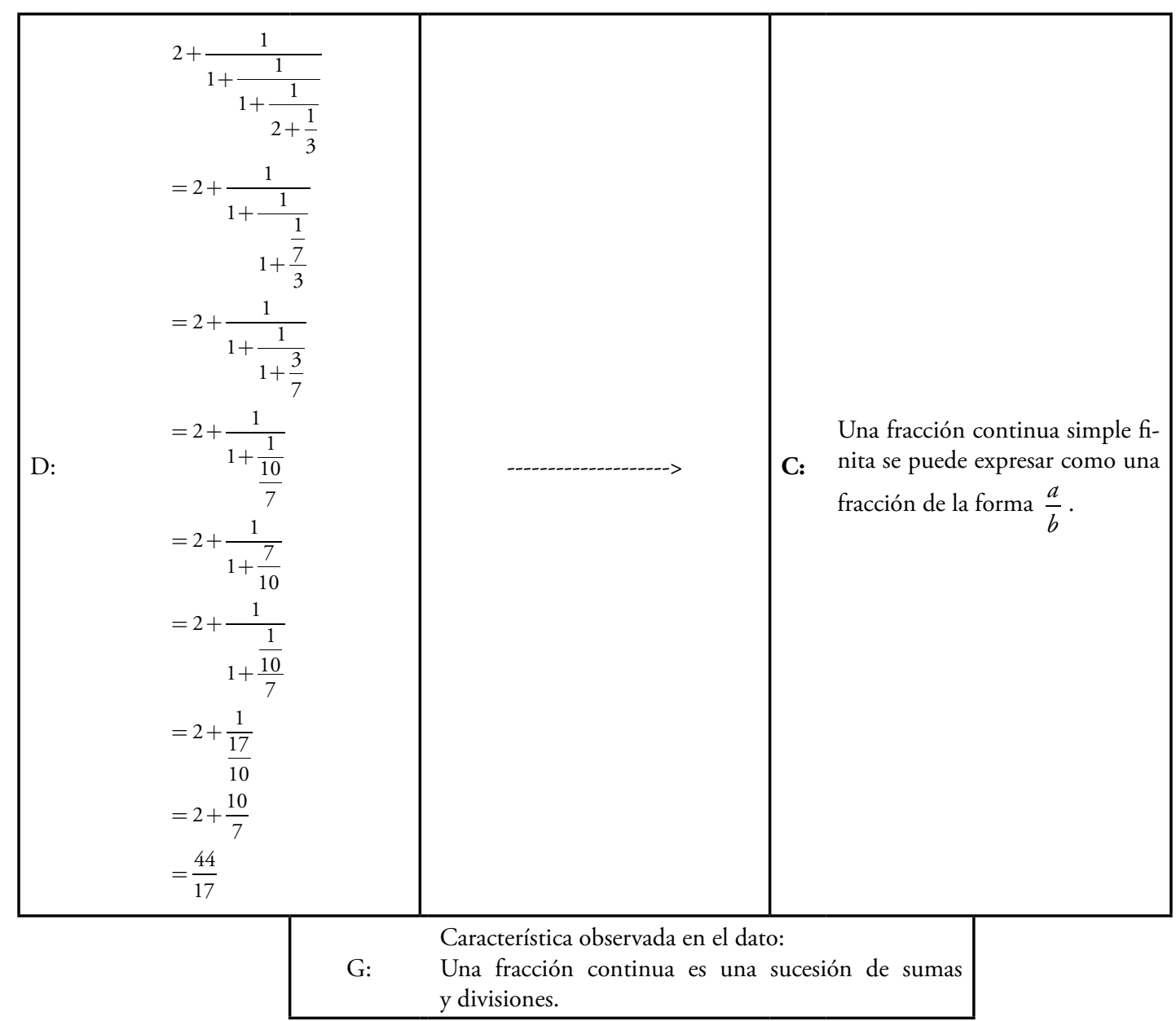

Fig. 18. Abducción con base en un caso particular en la tarea "Fraccionarios" 


\section{ABDUCCIÓN CON BASE EN RELACIONES OBSERVADAS EN UN DIAGRAMA GEOMÉTRICO}

El último tipo de conjetura definido por Cañadas y sus colaboradores (basadas en la percepción) es aquella conjetura que se establece a partir de la representación visual de un problema. La naturaleza de la representación permite reproducir las relaciones matemáticas existentes entre los elementos presentes en el problema y elaborar la conjetura. En términos de la teoría de Peirce, este tipo de abducción permite que se elabore una conjetura con base en las relaciones matemáticas representadas en un diagrama geométrico elaborado para representar una situación determinada. En la figura 19 se encuentran las partes que caracterizan a este tipo de abducción de acuerdo con el modelo de Toulmin.

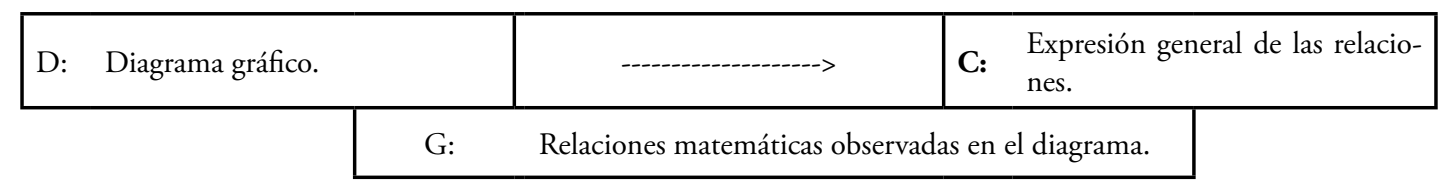

Fig. 19. Abducción con base en un diagrama geométrico

\section{Ejemplo en la tarea "Fracciones a racionales"}

Los estudiantes tenían como tarea establecer relaciones entre las posiciones y las fracciones. Al observar la gráfica encuentran regularidades en ciertas diagonales determinadas por las flechas; se construye el razonamiento presentado en la figura 20 .

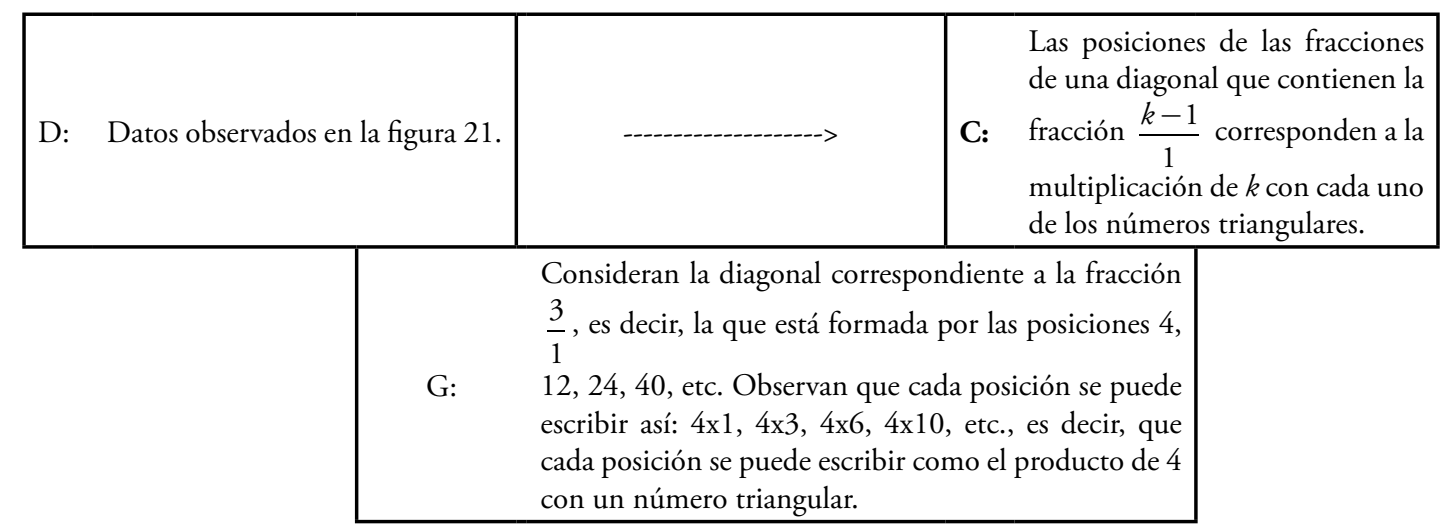

Fig. 20. Abducción con base en un diagrama geométrico en la tarea "Fracciones a racionales" 


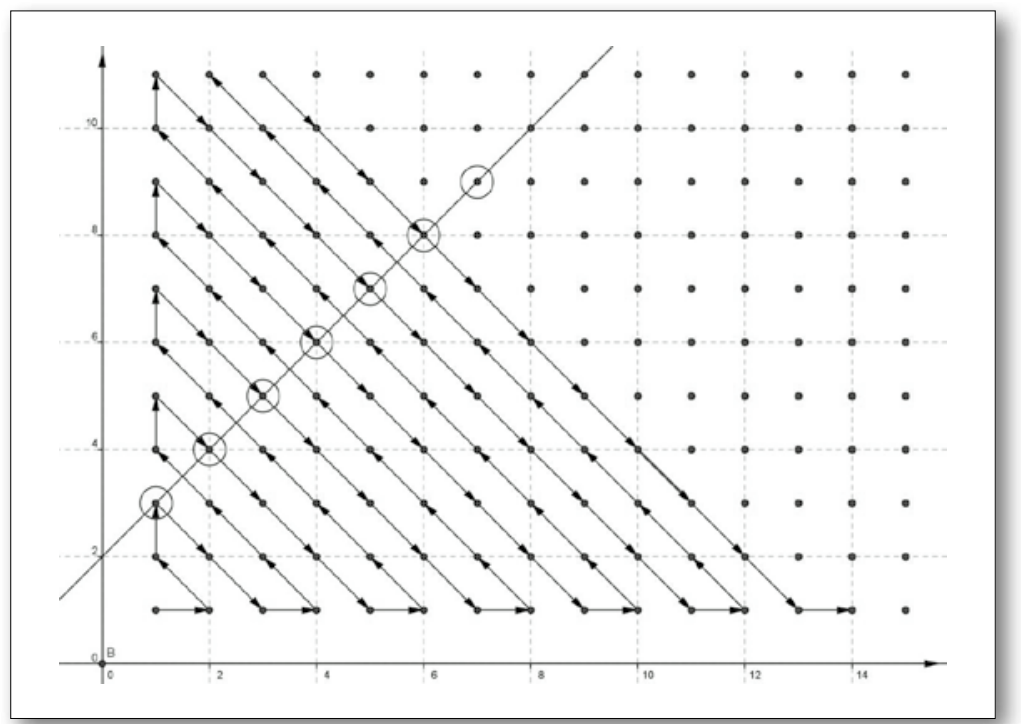

Fig. 21. Diagonal elegida por los estudiantes para ser estudiada

\section{Ejemplo en la tarea "Fraccionarios"}

En esta actividad los estudiantes transitaban entre diferentes representaciones de las fracciones. En particular, uno de los argumentos desarrollados por los alumnos generalizaba las relaciones entre la representación gráfica de las fracciones continuas y la representación numérica (ver figura 22).

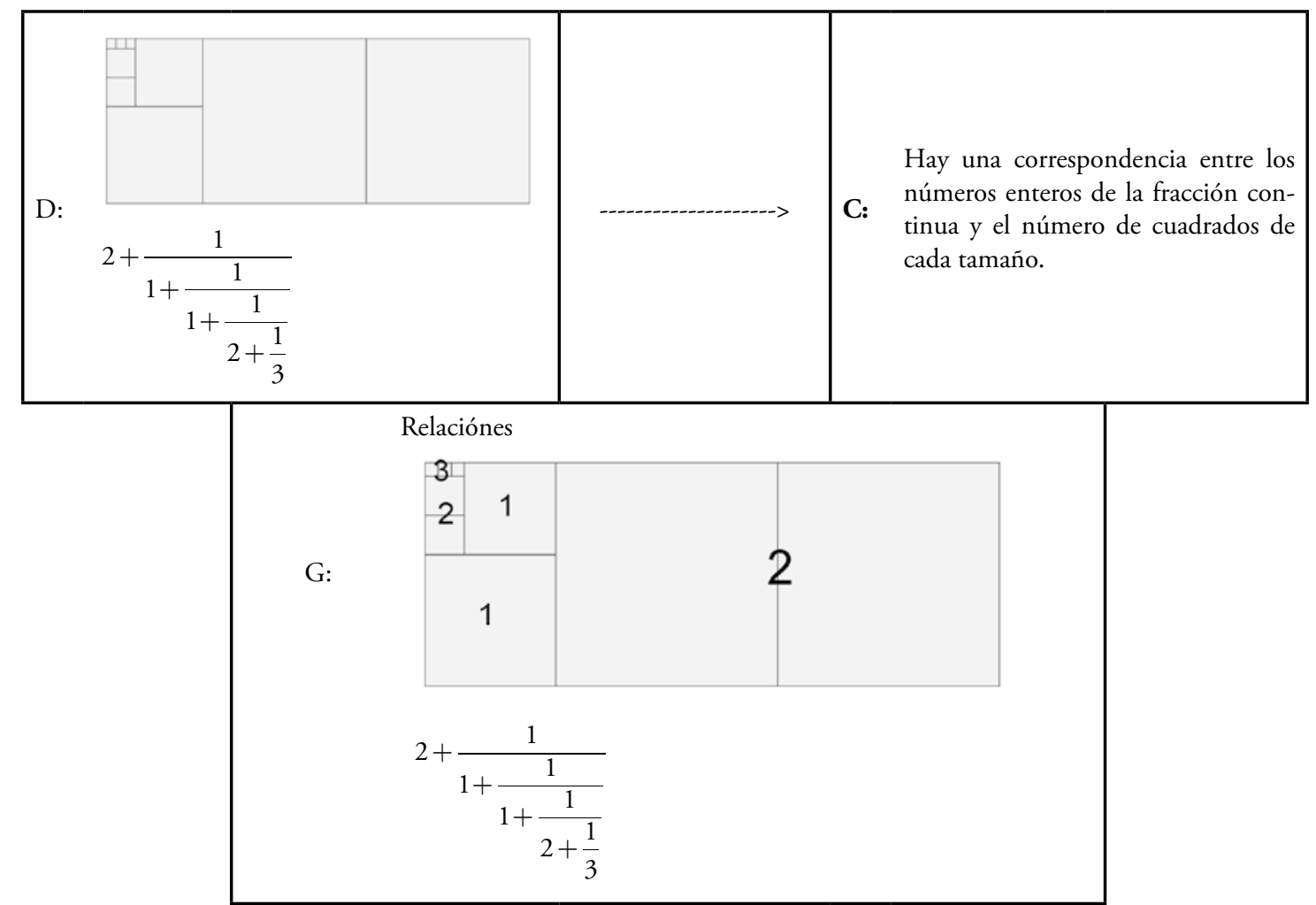

Fig. 22. Abducción con base en un diagrama geométrico en la tarea "Fraccionarios" 


\section{RAZONAMIENTO INDUCTIVO-VERIFICACIÓN EXPERIMENTAL DE LA CONJETURA}

\section{Ejemplo en la tarea "Suma y multiplicación de fracciones"}

Se observa este tipo de razonamiento cuando los estudiantes aceptan que el procedimiento gráfico creado para multiplicar fracciones es compatible con el método numérico usual, partiendo de unos ejemplos que presentan. La figura 23 presenta este tipo de razonamiento.

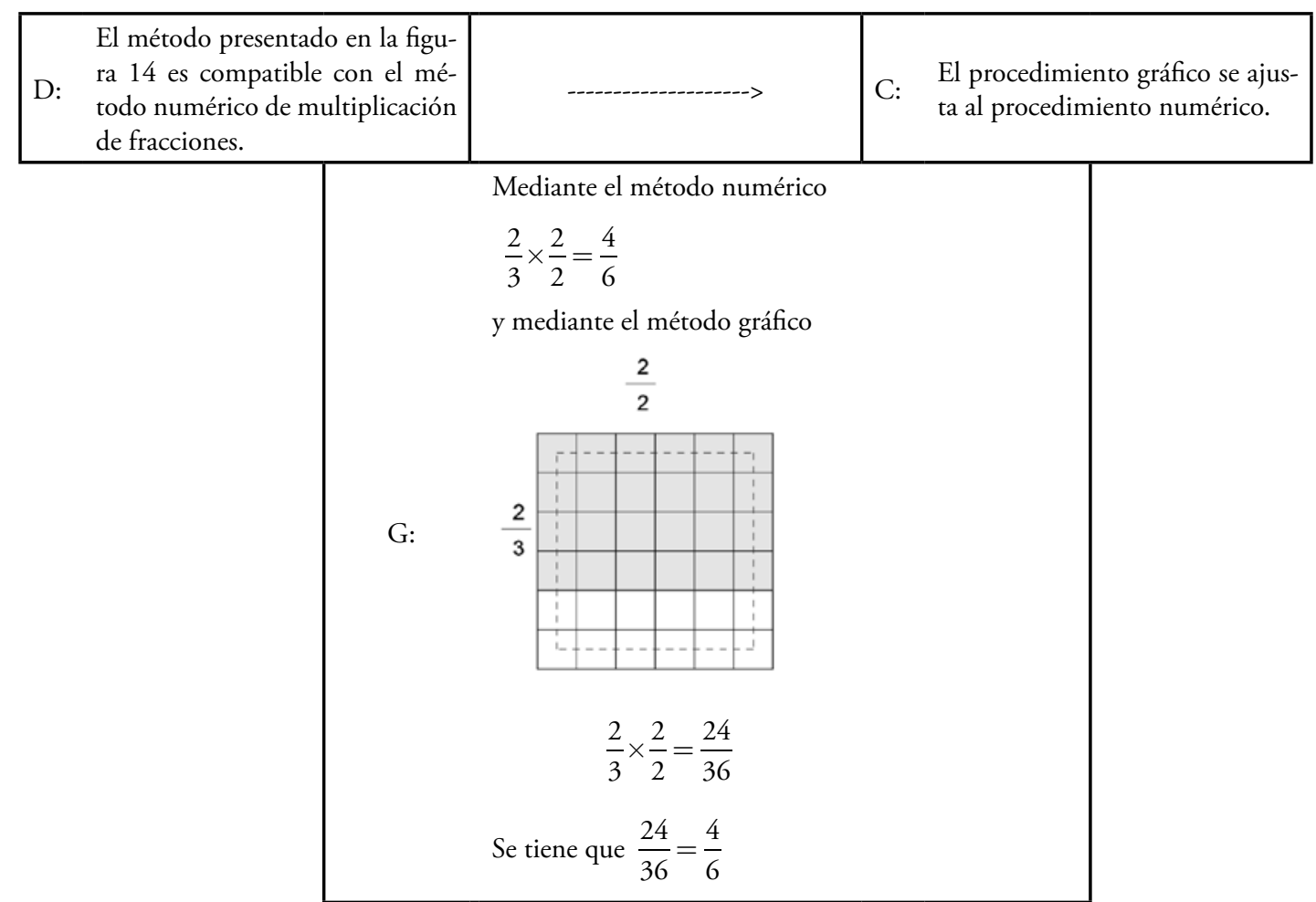

Fig. 23. Razonamiento inductivo generado en el desarrollo de la tarea "Suma y multiplicación de fracciones"

\section{RAZONAMIENTO DEDUCTIVO-VALIDACIÓN FORMAL DE LA CONJETURA}

El razonamiento deductivo se presenta en el desarrollo de argumentos que implican la aplicación de conceptos, procedimientos o reglas de inferencia. Estos elementos forman parte del garante del argumento y permiten que las conclusiones se obtengan de manera necesaria a partir de los datos.

En lo que sigue se presenta una clasificación de los argumentos deductivos observados en las tareas mencionadas. La clasificación de estos razonamientos obedece al tipo de garante o respaldo usados.

\section{DEDUCCIÓN A PARTIR DE UNA REGLA PREVIAMENTE ESTABLECIDA}

El razonamiento deductivo surge por la aplicación de una regla establecida previamente en el desarrollo de una tarea que se está realizando. El estudiante asume esa regla como válida y la aplica en casos particulares. En la figura 24 se presenta el esquema general de un argumento de este tipo. 


\begin{tabular}{|l|c|c|c|c|}
\hline D: Casos particulares. & \multicolumn{1}{|l|}{$\begin{array}{l}\text { Regla aplicada a los casos parti- } \\
\text { culares. }\end{array}$} \\
\hline \multirow{2}{*}{$\mathrm{G}: \quad \begin{array}{l}\text { Regla asumida como válida. } \\
\text { Datos adicionales. }\end{array}$} & \\
\cline { 2 - 3 } & $\mathrm{R}: \quad \forall x(P(x) \rightarrow Q(x))$ \\
\hline
\end{tabular}

Fig. 24. Esquema del razonamiento deductivo tipo "Deducción a partir de una regla previamente establecida”

\section{Ejemplo en la tarea "Epicicloides e hipocicloides"}

En la resolución de la actividad sobre ruletas cicloidales ${ }^{4}$ (ver Manrique, 2012) los alumnos establecieron mediante una abducción de patrones que para cualquier ruleta con directriz de radio $a$ y generatriz de radio $b$ se cumplía que $\frac{a}{b}=\frac{v}{r}$ (donde $v$ : vértices y $r$ : recorridos ). En el siguiente razonamiento presentado en la figura 25, los estudiantes usan esta regla para establecer si es posible que una ruleta exista bajo ciertas condiciones.

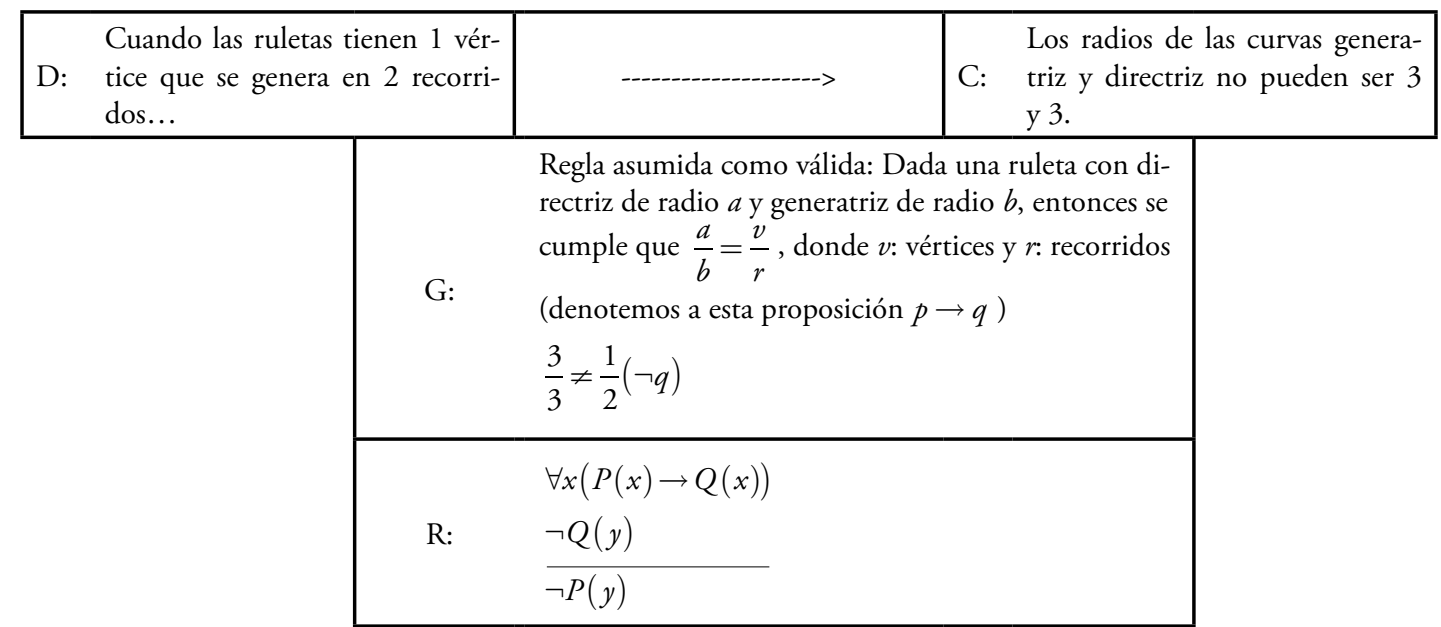

Fig. 25. Deducción a partir de una regla establecida previamente en la tarea "Epicicloides e hipocicloides"

\section{Ejemplo en la tarea "Racionales e irracionales"}

La tarea "Racionales e irracionales" consistió inicialmente en estudiar la secuencia presentada en la tabla 4 y describir la forma de los números que eran quitados del intervalo $(1,2)$ al hacer la intersección de todos los intervalos que aparecen. Tras esto se pidió a los estudiantes describir los números que se eliminan del mismo intervalo pero haciendo divisiones de los intervalos ya no solamente por dos, como en el caso de la tabla presentada, sino por tres, cuatro, cinco y todos los demás números naturales.

4. Si una curva rueda, sin resbalar, sobre otra curva fija, cualquier punto o segmento vinculado a la curva que gira describe una ruleta. Se denomina directriz a la curva fija, generatriz a la curva que rueda y polo al punto o segmento que describe la ruleta. Una ruleta cicloidal es una ruleta en la que la curva generatriz es una circunferencia. 
Tabla 4.

Secuencia utilizada en la tarea "Racionales e irracionales"

\begin{tabular}{|c|c|}
\hline Fila No. & Intervalos \\
\hline 1 & $\mathrm{I}_{11}=(1,2)$ \\
\hline 2 & $\mathrm{I}_{21}=\left(1, \frac{3}{2}\right) \quad \mathrm{I}_{22}=\left(\frac{3}{2}, 2\right)$ \\
\hline 3 & $\mathrm{I}_{31}=\left(1, \frac{5}{4}\right) \quad \mathrm{I}_{32}=\left(\frac{5}{4}, \frac{3}{2}\right) \quad \mathrm{I}_{33}=\left(\frac{3}{2}, \frac{7}{4}\right) \quad \mathrm{I}_{34}=\left(\frac{7}{4}, 2\right)$ \\
\hline 4 & $\mathrm{I}_{41}=\left(1, \frac{9}{8}\right) \quad \mathrm{I}_{42}=\left(\frac{9}{8}, \frac{5}{4}\right) \quad \mathrm{I}_{43}=\left(\frac{5}{4}, \frac{11}{8}\right) \quad \mathrm{I}_{44}=\left(\frac{11}{8}, \frac{3}{2}\right)$ \\
\hline$\ldots$ & $\mathrm{I}_{45}=\left(\frac{3}{2}, \frac{13}{8}\right) \quad \mathrm{I}_{46}=\left(\frac{13}{8}, \frac{7}{4}\right) \quad \mathrm{I}_{47}=\left(\frac{7}{4}, \frac{15}{8}\right) \quad \mathrm{I}_{48}=\left(\frac{15}{8}, 2\right)$ \\
\hline
\end{tabular}

Al realizar esta tarea, los estudiantes establecieron que los números de la forma $\frac{m^{n}+k}{m^{n}}$, con $1 \leq k \leq m^{n}-1$ desaparecen del intervalo $(1,2)$ después de hacer la intersección de todos los intervalos mencionados. Tras establecer esta regla, la usaron para evaluar algunos números y decidir si desaparecían o no del intervalo. Se desarrolla el razonamiento presentado en la figura 26.

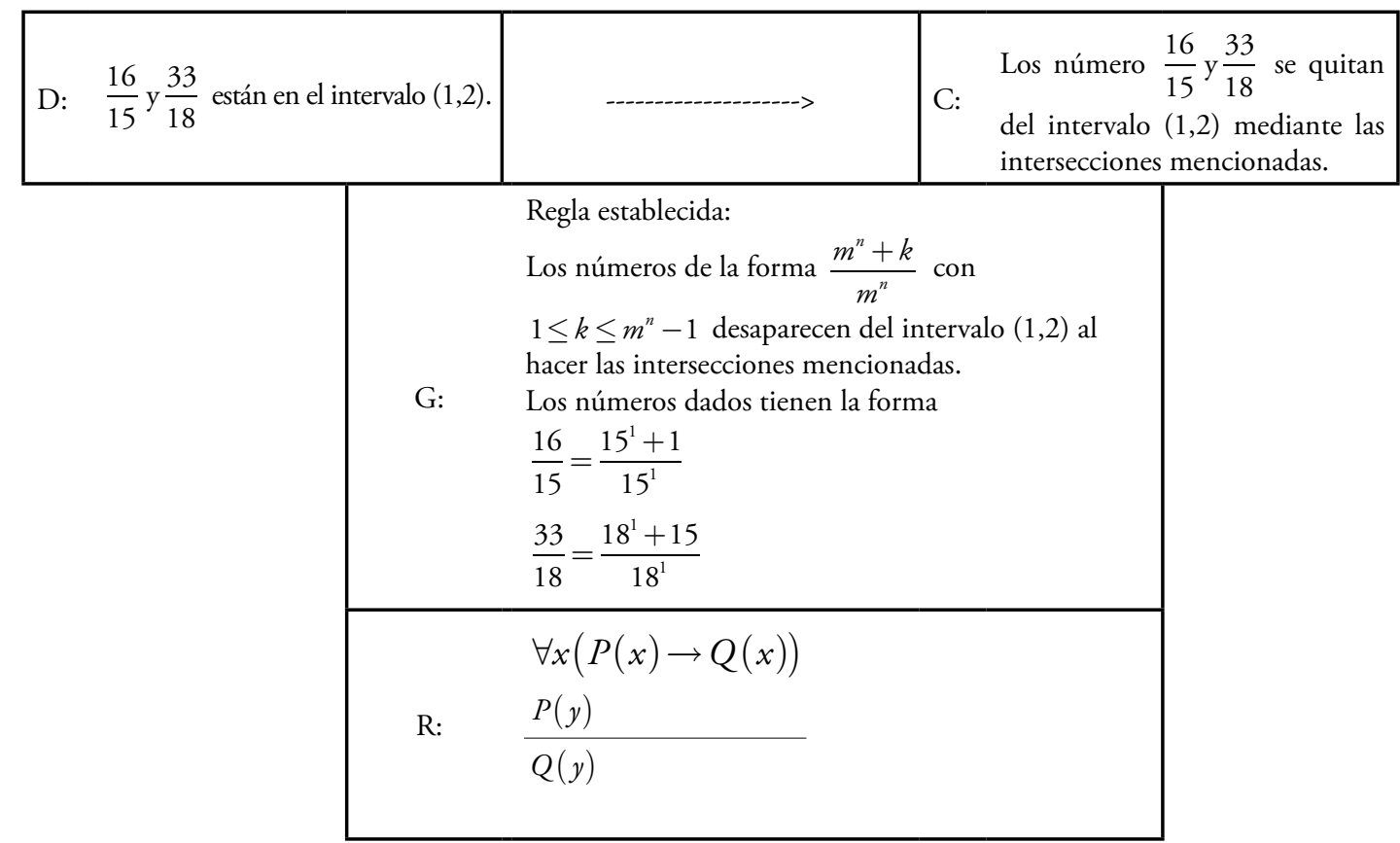

Fig. 26. Deducción a partir de una regla establecida previamente en la tarea "Racionales e irracionales" 


\section{Ejemplo en la tarea "Fraccionarios"}

Romero y Parra (2011) definen la forma de razonar deducción-imaginación, en la que se hace referencia a una regla creada previamente y se ajusta a la tipología que estamos definiendo. En el ejemplo que presentamos, los estudiantes habían establecido que "una fracción continua simple finita se puede operar y obtener con esta una fracción de la forma" y aplican esta regla en casos particulares. El razonamiento desarrollado se presenta en la figura 27.

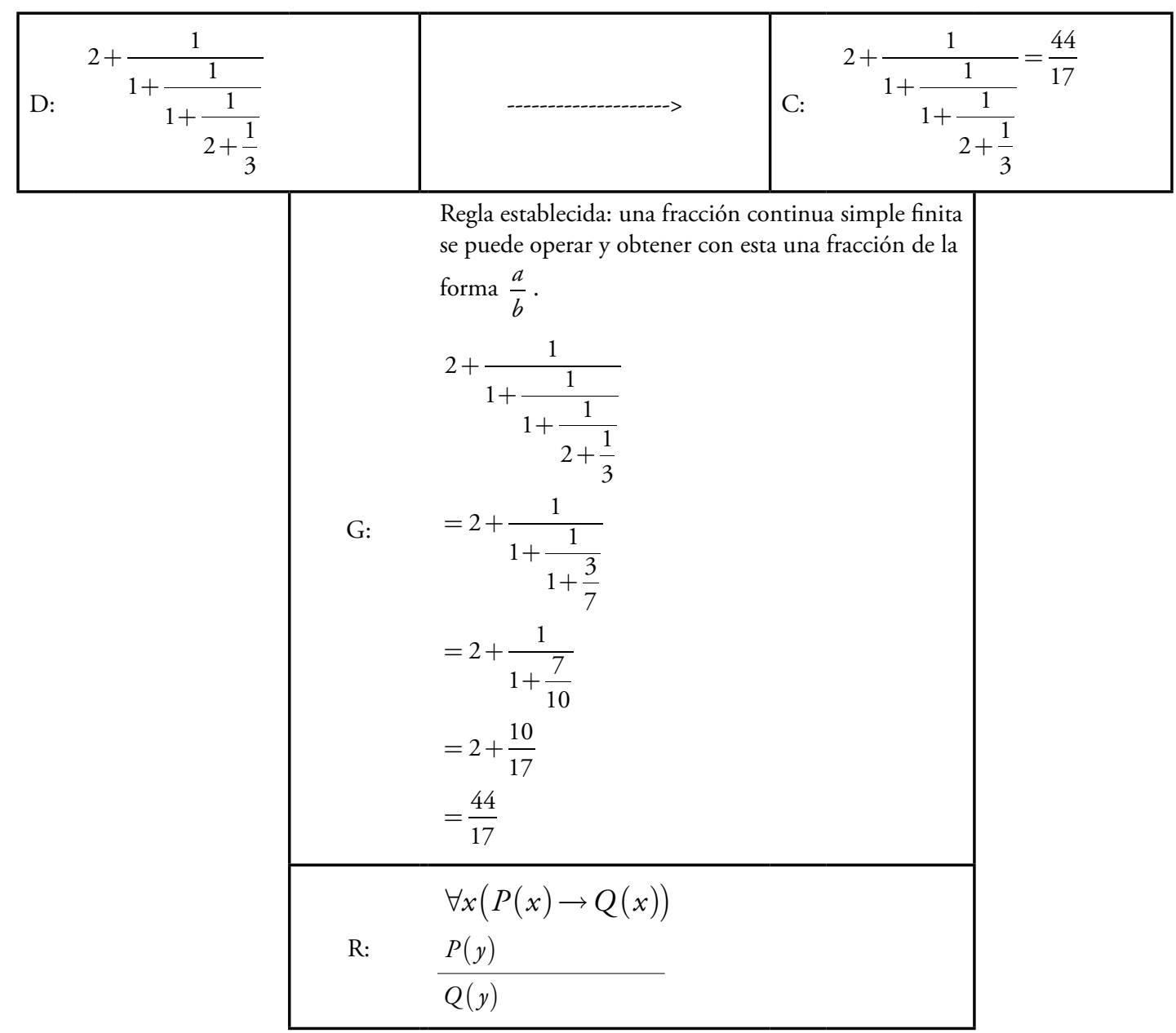

Fig. 27. Deducción a partir de una regla establecida previamente en la tarea "Fraccionarios"

\section{Contraejemplos}

En los desarrollos de las tareas propuestas a los estudiantes se encontraron razonamientos de este tipo. La estructura de un razonamiento de este tipo se presenta en la figura 28. 


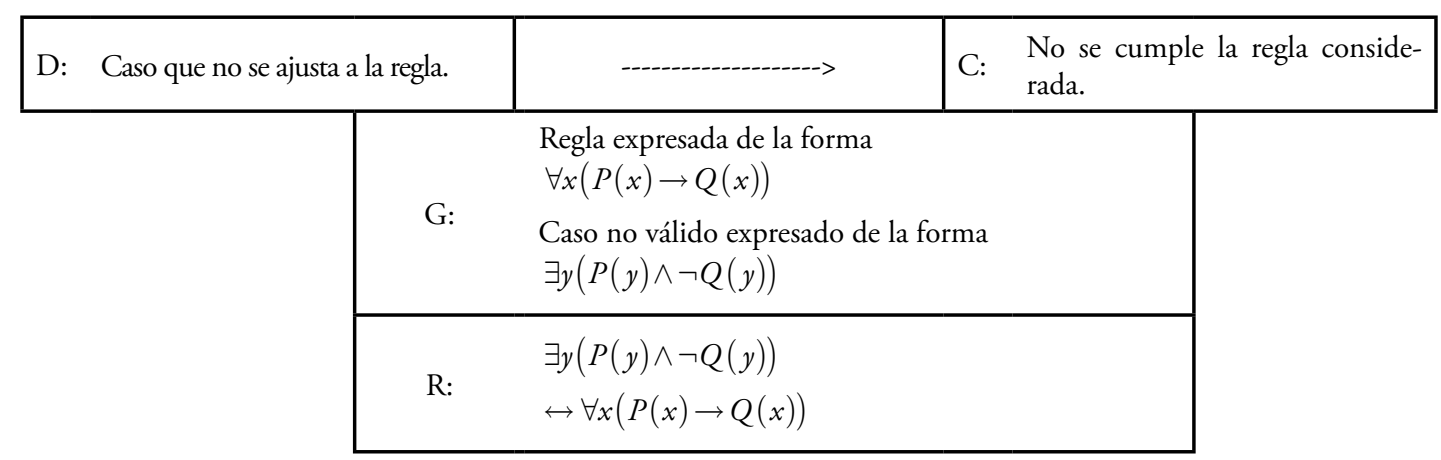

Fig. 28. Estructura razonamiento tipo "Contraejemplo"

\section{Ejemplo en la tarea "Fracciones a racionales"}

Los estudiantes habían establecido una regla asumiendo que la posición de las fracciones de la forma $\frac{a}{1}$ era el triangular $T_{a}$. En la figura 29 se presenta el razonamiento logrado por ellos para invalidar dicha regla.

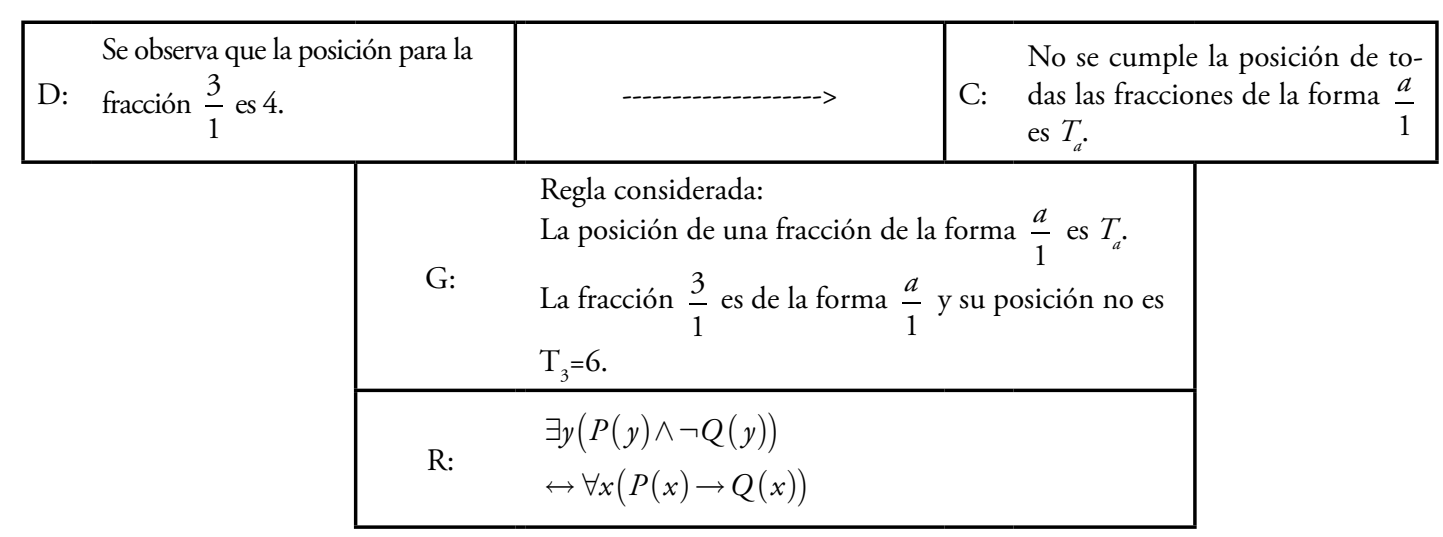

Fig. 29. Contraejemplo en la tarea "Fracciones a racionales"

\section{Ejemplo en la tarea "Racionales e irracionales"}

Los estudiantes, al comenzar el desarrollo de esta tarea, presentaron una forma general para describir los números que se retiraban del intervalo $(1,2)$ al realizar el procedimiento señalado anteriormente. La figura 30 presenta un contraejemplo descartando dicha forma general. 


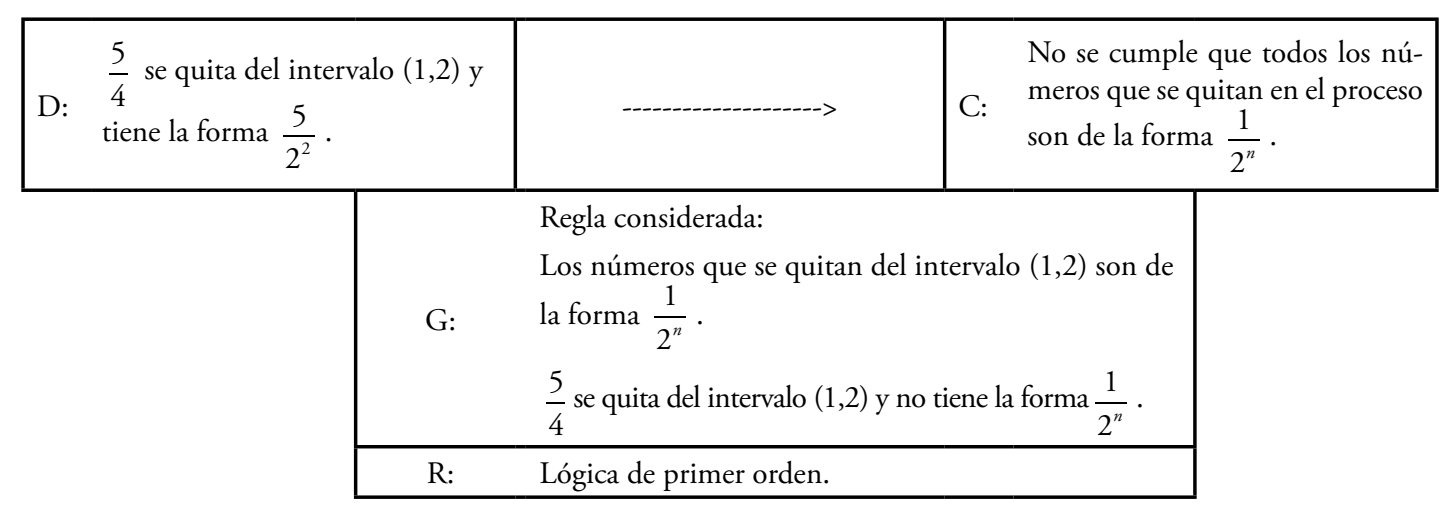

Fig. 30. Deducción tipo "Contraejemplo"

\section{TRANSFORMACIÓN DE DIAGRAMAS}

Mediante este tipo de deducción se transforma un diagrama geométrico, numérico o algebraico usando conceptos o procedimientos válidos en el estado hipotético de las cosas que se esté trabajando (geometría, aritmética, algebra). La clase de garante que se use obedece a la naturaleza del diagrama que actúe como datos del argumento: numérico, algebraico o geométrico. El esquema de este tipo de razonamiento se presenta en la figura 31 .

\begin{tabular}{|l|l|l|l|}
\hline D: Diagrama inicial. & C: Diagrama transformado. \\
\hline & G: & Hechos del contexto matemático considerado. & \multirow{2}{*}{} \\
\cline { 2 - 3 } & R: & Contexto matemático considerado. \\
\hline
\end{tabular}

Fig. 31. Razonamiento deductivo tipo "Transformación de diagramas"

\section{Ejemplo en la tarea "Racionales e irracionales"}

El razonamiento presentado en la figura 32 tiene lugar cuando los estudiantes y la profesora quieren garantizar que, con las intersecciones realizadas, se quitan los racionales del intervalo $(1,2)$.

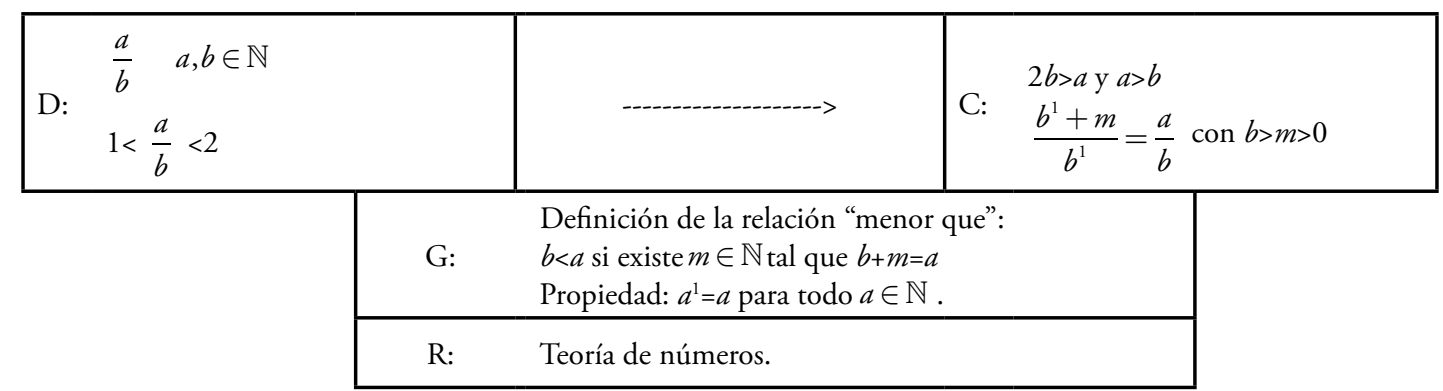

Fig. 32. Razonamiento deductivo tipo "Transformación de diagramas" 


\section{Ejemplo en la tarea "Algoritmo de la división"}

En esta tarea se presentan a los estudiantes divisiones de números $n$-males, ${ }^{5}$ y para cada una de las divisiones se solicita una descomposición del dividendo en ciertas sumas de grupos de potencias de diez. Se pide a los estudiantes que justifiquen por qué los dígitos de cociente de la división coinciden con los coeficientes de la descomposición. El enunciado de la tarea se presenta en la tabla 5.

Tabla 5.

Tabla que diligenciar en la tarea "Algoritmo de la división"

\begin{tabular}{|r|l|r|l|l|l|}
\hline Número & \multicolumn{1}{|c|}{ Base } & $\mathrm{k}$ & El número en la forma canónica & Número haciendo grupos de $\mathrm{k} 10^{\text {algo }}$ & Número $\mathrm{k}$ \\
\hline 1150 & Seis & 3 & & & \\
\hline 110203 & Cuatro & 11 & & & \\
\hline 423 & Cinco & 3 & & & \\
\hline 12236 & Siete & 5 & & & \\
\hline
\end{tabular}

El razonamiento presentado en la figura 33 se desarrolla cuando los estudiantes llevan a cabo una demostración de las conjeturas desarrolladas previamente. Para ello, expresan la división por medio de un diagrama diferente al usual y en el que se combinan dos formas de escribir los naturales: el diagrama cociente y la representación de naturales en un sistema de base (léase uno cero). Como conclusión se obtiene una transformación del diagrama usando propiedades de los números naturales.

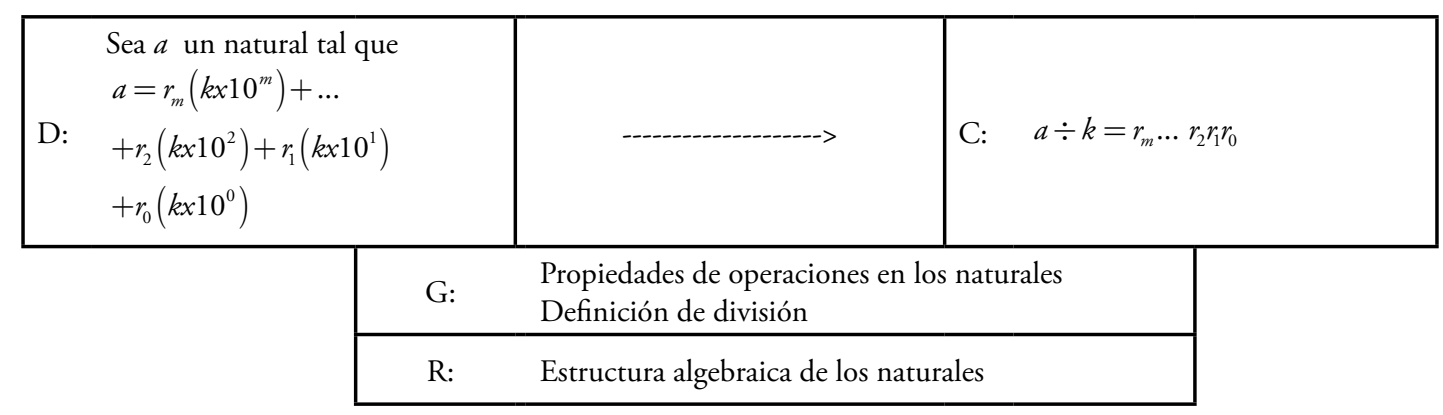

Fig. 33. Razonamiento deductivo tipo "Transformación de diagramas"

\section{CONCLUSIONES}

Si utilizamos el modelo propuesto anteriormente sobre razonamiento abductivo para presentar algunas conclusiones de este artículo, podríamos empezar por plantear que el proceso de descubrimiento matemático en las clases de matemáticas, particularmente aquellas que son para profesores de matemáticas, se caracteriza por el desarrollo de tres tipos de razonamiento: abductivo, deductivo e inductivo. Esta conclusión se sustenta en los resultados obtenidos de la actividad matemática lograda por los estudiantes al realizar las tareas propuestas en la investigación referida y los trabajos de grado realizados.

5. Un número $n$-mal es la representación de un número real en base $n$, es decir, si es un real, la expresión $n$-mal de $a$ es $a_{k} \times n^{k}+a_{k-1} \times n^{k-1}+\ldots+a_{0} \times n^{0}+a_{-1} \times n^{-1}+a_{-2} \times n^{-2}+\ldots=a_{k} a_{k-1} \ldots a_{0}, a_{-1}, a_{-2} \ldots$, donde $k$ es un número natural. 
En los ejemplos estudiados se observa que la estructura propuesta por Peirce para el desarrollo del método científico es útil en la descripción del descubrimiento matemático en la clase de matemáticas, la cual se caracteriza por la elaboración de conjeturas, la experimentación y la validación desarrolladas a través de diagramas matemáticos, como el geométrico, algebraico, tabular y el lenguaje natural escrito. Se observa además que estas formas de razonar permiten el tránsito entre diferentes formas de representar objetos matemáticos, lo que amplía la actividad matemática realizada por los estudiantes.

Otra conclusión que se puede plantear es que los tres tipos de razonamiento propuestos por Peirce pueden ajustarse al modelo de Toulmin sobre argumentación. Estos tres tipos de razonamiento son esencialmente distintos: el primero, el abductivo, se relaciona con la capacidad de crear, observar, encontrar relaciones, hacer analogías y establecer conjeturas. El segundo tipo de razonamiento se relaciona con la experimentación, con la revisión en detalle de las conjeturas propuestas, la cual permite ajustarlas o reevaluarlas según cada caso. El tercer tipo de argumento, el deductivo, garantiza conclusiones necesarias, conclusiones que son difíciles de refutar.

En relación con el razonamiento abductivo, podemos afirmar que este se caracteriza, a partir del modelo de Toulmin, por que sus conclusiones corresponden a conjeturas y los garantes a aquello que es común en los datos, por ejemplo, patrones, relaciones, regularidades, propiedades, entre otros. Estos garantes permiten clasificar los argumentos en diferentes tipos; adicionalmente, las diferentes formas en que este tipo de razonamiento se presenta obedecen al tipo de diagrama que actúa como datos del argumento, lo cual a su vez incide en el tipo de garante usado. Así, por ejemplo, cuando los datos se componen de un conjunto de diagramas numéricos, el garante corresponde a patrones observados en estos diagramas, mientras que si se tratan diagramas geométricos, el garante se constituye a partir de las relaciones observadas en dicho diagrama.

Respecto al razonamiento deductivo, según el modelo de Toulmin, este se caracteriza por pasar del antecedente de la conjetura, al consecuente por medio de reglas establecidas previamente, reglas de inferencia lógica, conjeturas realizadas anteriormente, teoremas, axiomas, entre otros. Este razonamiento es de varios tipos; el más usado por los estudiantes es el contraejemplo, que es empleado cuando intentan determinar la no validez de una conjetura. En los razonamientos deductivos obtenidos de la aplicación de una regla, se observa que algunos de los garantes no se han validado previamente; en muchas ocasiones corresponden a hipótesis que se construyen con razonamientos abductivos no validados.

El razonamiento inductivo es uno de los más usados por los estudiantes; en muchas ocasiones, este se aplica como único medio de validación de conjeturas. Esto porque los estudiantes no encuentran necesario probar la validez de las conjeturas. Por ejemplo, cuando se utilizan aplicativos de matemática dinámica, los estudiantes encuentran un sinnúmero de ejemplos donde su conjetura se valida, por lo que no ven necesario realizar una prueba.

Consideramos que el desarrollo de las formas de razonar depende del tipo de tarea que se propone al estudiante. Algunas de estas permiten la elaboración de múltiples conjeturas y otras propenden por el desarrollo del razonamiento deductivo, fortaleciendo las acciones de validación. Este aspecto puede ser motivo de atención en futuras investigaciones.

Respecto a la tipología propuesta por Cañadas et al. (2008), se puede concluir lo siguiente:

En la abducción con diagramas dinámicos, se observa que, además de las propiedades invariantes, en los entornos dinámicos se pueden encontrar otros garantes que permiten la formulación de conjeturas, entre los que se encuentran: relaciones entre objetos geométricos, dependencia o independencia entre variables y cambios entre los sistemas de representación de los objetos matemáticos presentados.

Los datos y los garantes usados en la abducción de patrones, además de lo presentado por Cañadas et al. (2008), se observa que pueden presentarse en diferentes registros de representación, como el tabular o aquellos generados por entornos de matemática dinámica. 
Finalmente, cabe señalar que, si bien coincidimos con los pasos propuestos por estos autores en el proceso de construcción y análisis de una conjetura, es importante distinguir el papel que tiene la conjetura en cada uno de estos momentos, lo cual tiene que ver directamente con el tipo de razonamiento que se desarrolla en cada uno. De esta manera, consideramos que todos los razonamientos abductivos incluyen en su conclusión la conjetura y se desarrollan en los pasos de construcción de esta (observación de casos, búsqueda de patrones, regularidades o relaciones, formulación de una conjetura). Los razonamientos inductivos incluyen la conjetura en sus datos y permiten la validación de esta en casos particulares; tal y como señalan estos autores, este tipo de validación no es suficiente para realizar una generalización. Para esto es necesario desarrollar un razonamiento deductivo, en el cual la conjetura actúa como garante.

\section{REFERENCIAS}

Campos, D. (2010). The imagination and hypotesis-making in mathematics: A Peirce account. In M. Moore (ed.). New Essays on Peirce's Mathematical Philosophy. Illinois: Open Court.

Cañadas, M. C. y Castro, E. (2007). A proposal of categorisation for analysing inductive reasoning. PNA, 1(2), pp. 67-78.

Cañadas, M. C.; Deulofeu, J.; Figueras, L.; Reid, D. y Yevdokimov, O. (2008). Perspectivas teóricas en el proceso de elaboración de conjeturas e implicaciones para la prática: tipos y pasos. Enseñanza de las ciencias, 26 (3), pp. 431-444.

Снамizo, J. (2007). Las aportaciones de Toulmin a la enseñanza de las Ciencias. Enseñanza de las ciencias, 25(1), pp. 136-146.

MANRiQue, V. (2012). Caracterización y clasificación de las formas de razonar usadas por un grupo de profesores en formación al resolver una actividad sobre ruletas cicloidales. Tesis de pregrado no publicada. Universidad Pedagógica Nacional, Bogotá, Colombia.

MEN. (1998). Lineamientos Curriculares de Matemáticas. Bogotá: Ministerio de Educación Nacional, Colombia.

Nitola, M. y Castaño, Y. (2011). Estudio de los tipos de razonamientos y habilidades matemáticas que utilizan algunos estudiantes del curso de Sistemas Numéricos de la Licenciatura en Matemáticas al realizar una actividad sobre enumerabilidad de las fracciones. Tesis de pregrado no publicada. Universidad Pedagógica Nacional, Bogotá, Colombia.

Pedemonte, B. (2003). ¿What kind of proof can be constructed following an abductive argumentation? Trabajo presentado en el Cerme 3, Bellaria, Italy.

Pedemonte, B. (2007). Structural relationships between argumentation and proof in solving open problems in algebra. Trabajo presentado en el Cerme 5, Larnaca, Chipre.

Pedemonte, B. y Reid, D. (2011). The role of abduction in proving processes. Educational studies in mathematics, 76(3), pp. 281-303.

http://dx.doi.org/10.1017/s10649-010-9275-0

Redondo, A. y H., J (2005). Fracciones continuas, números metálicos y sucesiones generalizadas de Fibonacci. Revista Suma, 50, pp. 53-63.

Romero, N. y Parra, Y. (2011). Identificación del razonamiento inductivo, abductivo y deductivo presente en las soluciones que algunos estudiantes dieron al resolver una actividad sobre fracciones continuas simples finitas en el espacio académico de sistemas numéricos de la licenciatura en matemáticas. Tesis de pregrado no publicada. Universidad Pedagógica Nacional, Bogotá, Colombia. 
Santaella, L. (2009). La evolución de los tres tipos de razonamiento: Abducción, inducción y deducción. Disponible en: <http://www.unav.es/gep/AN/Santaella.html>. (Última consulta: 28 de febrero de 2010).

Sardá, A. y Sanmartí, N. (2000). Enseñar a argumentar científicamente: Un reto de las clases de ciencias. Enseñanza de las ciencias, 18(3), pp. 405-422.

Toulmin, S. (2003). The uses of argument. Cambridge University Press.

http://dx.doi.org/10.1017/CBO9780511840005 


\title{
Discovery process in mathematics class: abductive, inductive and deductive reasoning
}

\author{
María Nubia Soler-Álvarez \\ Universidad Pedagógica Nacional \\ nsoler@pedagogica.edu.co \\ Viviana Helena Manrique \\ vmanriqueperez@gmail.com
}

This article stems from the research "DMA-204-10: Actividades Matemáticas para el desarrollo de procesos lógicos: los razonamientos inductivo y abductivo" developed in Colombia at the Universidad Pedagógica Nacional in 2010 and 2011. We characterized different ways of reasoning used by a group of training teachers when solving tasks related to rational and irrational numbers.

The theoretical approach used for the development of the research links three different theories: Peirce's theory about scientific method and mathematical reasoning, Toulmin's model of argument and the theoretical perspective suggested by Cañadas, Deulofeu, Figueiras, Reid and Yevdokimov (2008) in relation with the process of formulation and validation of conjectures. With the relations established between these perspectives, we describe the process of discovery in the mathematics class and interpret the argumentations developed by the students. Toulmin model has three components: data, warrant and assertion; warrant allows transition between data and assertion.

The process of discovery in the mathematics class is characterized by the three types of reasoning mentioned by Peirce: students formulate conjectures, reasoning of abductive form; they check conjectures experimenting on mathematical diagrams, reasoning of inductive form, and they validate their conjectures using deductive reasoning. We observed, besides, the fact that the development of these types of reasoning allows for the transition among representations of mathematical objects.

First, reasoning relates to the ability to create, observe, find relations, and establish analogies and conjectures. Warrant of this type of reasoning appears from what is common in the information observed, for example, patterns, relations, regularities, properties, constants, among others. This type of reasoning appears in different forms. It depends on the diagram of information presented. These diagrams, in turn, affect the type of warrant used. For example, when the information consists of a set of numerical data, warrant corresponds to patterns observed. Whereas, if diagram is geometric, warrant is constituted from relations observed between objects observed.

In the mathematics classes observed, we identified five different types of abductive reasoning: Pattern's abduction, Abduction with dynamical graphs, Abduction through analogy, Abduction based on a characteristic observed in a particular case and Abduction based on the relations observed in a geometric graph.

Deductive reasoning refers to necessary inference. Warrants are, for example, rules, logical rules, conjectures, theorems, axioms, among others. In the classes observed, this reasoning is of three types: Deduction from a previously established rule, Counter examples and Transformation of diagrams. Counter example is used by students to prove that some conjecture is false. Warrants of a deductive reasoning obtained from the application of a rule are, sometimes, conjectures not validated. In many occasions, they are assertion that was constructed by abductive reasoning.

The inductive reasoning is often used by the students to become convinced of the validity of conjectures. Sometimes, this one applies like a single method of validation. It is because students do not need to prove their validity, for example, when they use diagrams presented in programs of dynamical mathematics, students find many examples where conjecture is true. For this reason, they do not need to do a proof.

The development of these ways of reasoning depends significantly on the type of tasks proposed to the students, some of which allow production of multiple conjectures and tend others for the development of deductive reasoning, strengthening the actions of validation.

\section{References}

Cañadas, M. C., Deulofeu, J., Figueras, L., Reid, D., \& Yevdokimov, O. (2008). Perspectivas teóricas en el proceso de elaboración de conjeturas e implicaciones para la práctica: tipos y pasos. Enseñanza de las ciencias, 26(3), 431-444. 